ARTICLE

https://doi.org/10.1038/s41467-019-09346-z

\title{
Asymmetric ephaptic inhibition between compartmentalized olfactory receptor neurons
}

\author{
Ye Zhang ${ }^{1}$, Tin Ki Tsang (10 ${ }^{1}$, Eric A. Bushong (1) ${ }^{2}$, Li-An Chu ${ }^{3}$, Ann-Shyn Chiang (1) ${ }^{3}$, Mark H. Ellisman², \\ Jürgen Reingruber ${ }^{4,5} \&$ Chih-Ying Su ${ }^{1}$
}

In the Drosophila antenna, different subtypes of olfactory receptor neurons (ORNs) housed in the same sensory hair (sensillum) can inhibit each other non-synaptically. However, the mechanisms underlying this underexplored form of lateral inhibition remain unclear. Here we use recordings from pairs of sensilla impaled by the same tungsten electrode to demonstrate that direct electrical ("ephaptic") interactions mediate lateral inhibition between ORNs. Intriguingly, within individual sensilla, we find that ephaptic lateral inhibition is asymmetric such that one ORN exerts greater influence onto its neighbor. Serial block-face scanning electron microscopy of genetically identified ORNs and circuit modeling indicate that asymmetric lateral inhibition reflects a surprisingly simple mechanism: the physically larger ORN in a pair corresponds to the dominant neuron in ephaptic interactions. Thus, morphometric differences between compartmentalized ORNs account for highly specialized inhibitory interactions that govern information processing at the earliest stages of olfactory coding.

\footnotetext{
${ }^{1}$ Neurobiology Section, Division of Biological Sciences, University of California San Diego, 9500 Gilman Drive, La Jolla, CA 92093, USA. ${ }^{2}$ National Center for Microscopy and Imaging Research, Center for Research in Biological Systems, University of California San Diego, 9500 Gilman Drive, La Jolla, CA 92093 , USA. ${ }^{3}$ Brain Research Center, National Tsing Hua University, No. 101, Section 2, Kuang-Fu Road, Hsinchu 30013, Taiwan. ${ }^{4}$ Institut of Biology, École Normale Supérieure (IBENS), 46 rue d'Ulm, 75005 Paris, France. ${ }^{5}$ INSERM U1024, 75005 Paris, France. These authors contributed equally: Ye Zhang, Tin Ki Tsang. Correspondence and requests for materials should be addressed to C.-Y.S. (email: c8su@ucsd.edu)
} 
onic fluxes from neuronal activity lead to changes in the extracellular potential ${ }^{1}$, which can influence the excitability of adjacent neurons by electrical field effects, known as ephaptic interaction ${ }^{2,3}$. First observed between two axons brought together experimentally ${ }^{4,5}$, ephaptic interaction takes place between uninsulated neuronal processes packed into an electrically isolated microenvironment ${ }^{2,3}$. Such an arrangement commonly occurs in fascicles containing bundles of unmyelinated axons, such as the mammalian olfactory nerve ${ }^{6}$ and the interoceptive sensory system ${ }^{7}$, as well as in regions of the nervous system including the fish hindbrain, mammalian cerebellum, hippocampus, and retina ${ }^{1-3,8,9}$. Despite their likely prevalence, field effects have long been considered nebulous ${ }^{10}$, as most neurons that are known to interact ephaptically also communicate via chemical synapses or gap junctions ${ }^{2,3,8}$. In addition, ephaptic interaction is notoriously difficult to study because it is enabled by high extracellular resistance and density of neural membranes ${ }^{2,3}$, none of which are amenable to in vivo experimental manipulation. Therefore, it remains unclear whether and how ephaptic interaction by itself is sufficient to influence circuit function.

Taking advantage of the powerful genetic toolkit of Drosophila melanogaster, we showed that olfactory receptor neurons (ORNs) housed in the same sensory hair, or sensillum, can inhibit each other, and that such lateral inhibition can modulate odor-guided behavior $^{11}$. Despite the lack of direct synaptic connections, transient activation of one ORN rapidly suppresses the ongoing activity of its neighbor ${ }^{11}$. Electric circuit modeling suggested a potential mechanism for this nonsynaptic signaling ${ }^{11,12}$ : in the restrictive space of a sensillum lumen, the high resistance of the lymph ${ }^{13}$ favors the generation of field effects between compartmentalized $\mathrm{ORNs}^{2,3}$. However, whether ephaptic interaction underlies the inhibition between ORNs has not been directly demonstrated.

In addition, outstanding questions remain about the peripheral organization of ORNs. Most insect ORNs housed in the same sensillum exhibit distinct and characteristic extracellular spike amplitudes. Grouped ORNs are thus named "A", "B" or " $C$ " based on their relative spike amplitudes in descending order ${ }^{14}$. In fruitflies, olfactory sensilla contain up to four neurons, with the majority of them housing two ORNs ${ }^{14,15}$. Intriguingly, certain odorant receptors are exclusively expressed in the large-spike " $\mathrm{A}$ " neurons, whereas others in the small-spike ORNs. For instance, in D. melanogaster, the Or22a receptor is expressed in the largespike " $A$ " neuron in the antennal basiconic sensilla of type 3 (ab3A), which is paired with a small-spike neighbor expressing Or85b (ab3B) ${ }^{16-18}$. The ab3A(Or22a)-ab3B(Or85b) arrangement is also observed in other Drosophila species ${ }^{19,20}$. These evolutionarily conserved patterns of ORN arrangement point to functional constraints in neuronal organization. They also imply that grouped ORNs have distinguishable functional characteristics. However, beyond ligand specificity ${ }^{21}$, little is known about whether and how the large-spike "A" ORN functionally differs from its small-spike neighbor.

In this study, we use a novel experimental approach to provide direct experimental evidence that ephaptic coupling alone is sufficient to drive lateral inhibition between ORNs. In addition, combining electrophysiological recordings, morphometric analysis based on serial block-face scanning electron microscopy $(\mathrm{SBEM})^{22}$ and circuit modeling, we uncover a surprising functional disparity between compartmentalized ORNs in ephaptic inhibition and elucidate the underlying mechanism. Our study thus establishes the peripheral olfactory system of Drosophila as an ideal model to illuminate the impact of ephaptic interaction on circuit function and to determine its general operating principle.

\section{Results}

Direct evidence of ephaptic inhibition between ORNs. How does one prove that ORNs inhibit each other ephaptically if the inhibition is not mediated by any manipulatable target? We addressed this question by testing whether direct electrical interaction is sufficient to cause inhibition between ORNs. If lateral inhibition proceeds electrically in a sensillum, by means of experimental manipulation, we expect to observe similar cross inhibition between ORNs housed in different yet electrically coupled sensilla. We therefore performed extracellular recordings using a metal electrode to connect the electric fields of two adjacent sensilla, a manipulation that allows for direct demonstration of the impact of electrical interaction ${ }^{23}$.

In the control experiment examining an individual ab1 sensillum, the sustained spike responses of ab1A and ab1B to a prolonged dose of vinegar (large spikes in Fig. 1a, top panel, $\mathrm{ab} 1 \mathrm{~A}$ and $\mathrm{ab} 1 \mathrm{~B}$ spikes could not be sorted reliably and were thus grouped) were markedly reduced by a pulse of superimposed $\mathrm{CO}_{2}$ that activated ab1C (small spikes). The inhibition of ab1A/B by $\mathrm{CO}_{2}$ was abolished in mutant flies lacking functional $\mathrm{CO}_{2}$ receptors ${ }^{24}$ (Fig. 1a, bottom panel), indicating that the inhibition of $\mathrm{ab} 1 \mathrm{~A} / \mathrm{B}$ depends on the excitation of $\mathrm{ab} 1 \mathrm{C}$, consistent with our earlier results by genetic ablation of $a b 1 C^{11}$.

Next we used a tungsten electrode to impale two adjacent sensilla, ab1 and ab3, in order to connect their electric fields so that the field changes in one sensillum can be detected by ORNs in the other sensillum ${ }^{23}$. This approach eliminates the influence of other possible nonsynaptic mechanisms, such as inhibition mediated by shared odorant binding proteins ${ }^{25}$. Using this paradigm, we recorded ORN activity from both ab1 and ab3 sensilla simultaneously (Fig. 1b, top panel). As predicted, the sustained responses of ab1A/B were similarly inhibited by a superimposed pulse of E2-hexenol that excited the electrically coupled ab3B. The cross-inhibition depended on the electric coupling because withdrawing the tungsten electrode from the ab3 sensillum also abolished the inhibition of ab1A/B by E2hexenol (Fig. 1b, middle panel, from the same group of ab1A/B neurons). We note that vinegar did not elicit significant sustained responses in ab3A (Fig. 1b, bottom panel). Therefore, the inhibition observed in the bridged configuration (Fig. 1b, top panel) was driven by the activation of electrically coupled ab3B. In control experiments, we showed that the bridged configuration did not cause any change in the responses of individual ORNs (Supplementary Fig. 1).

Furthermore, when ab3A was chronically activated by optogenetic stimulation, the sustained response could also be inhibited by a pulse of $\mathrm{CO}_{2}$, which excited ab1C housed in the electrically coupled sensillum (Fig. 1c). Together, these results provide direct evidence that the sustained response of one ORN was cross-inhibited by the excitation of another ORN via the interconnected electric fields. In other words, direct electrical interaction, or ephaptic coupling, is sufficient to drive lateral inhibition between ORNs that share the same electric field.

Field response and ephaptic inhibition. We next asked whether the large-spike "A" ORNs are functionally distinguishable from their small-spike neighbors in ephaptic interaction. In earlier work, we showed that lateral inhibition between ORNs is bidirectional: Transient activation of the "B" ORN inhibits the sustained response of the " $A$ " neuron and vice versa ${ }^{11}$. However, it is unclear whether the bidirectional inhibition is equal in strength. To compare directly the strength of A-to-B and B-to-A inhibition is technically challenging because high frequency firing of the large-spike "A" neuron occludes spike activity of the "B" neuron. In addition, the degree of inhibition is influenced by the activity 
a
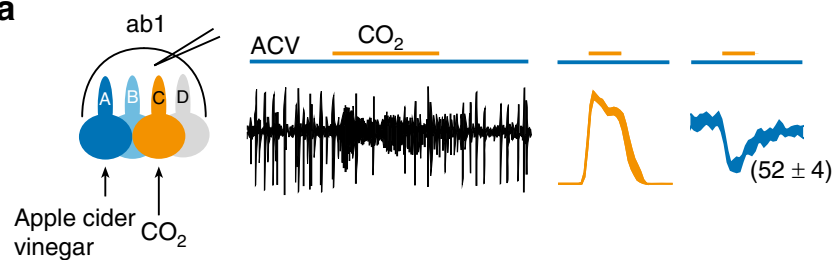

vinegar
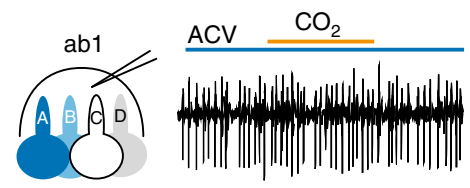

$0.5 \mathrm{~s}$

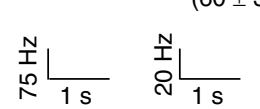

b
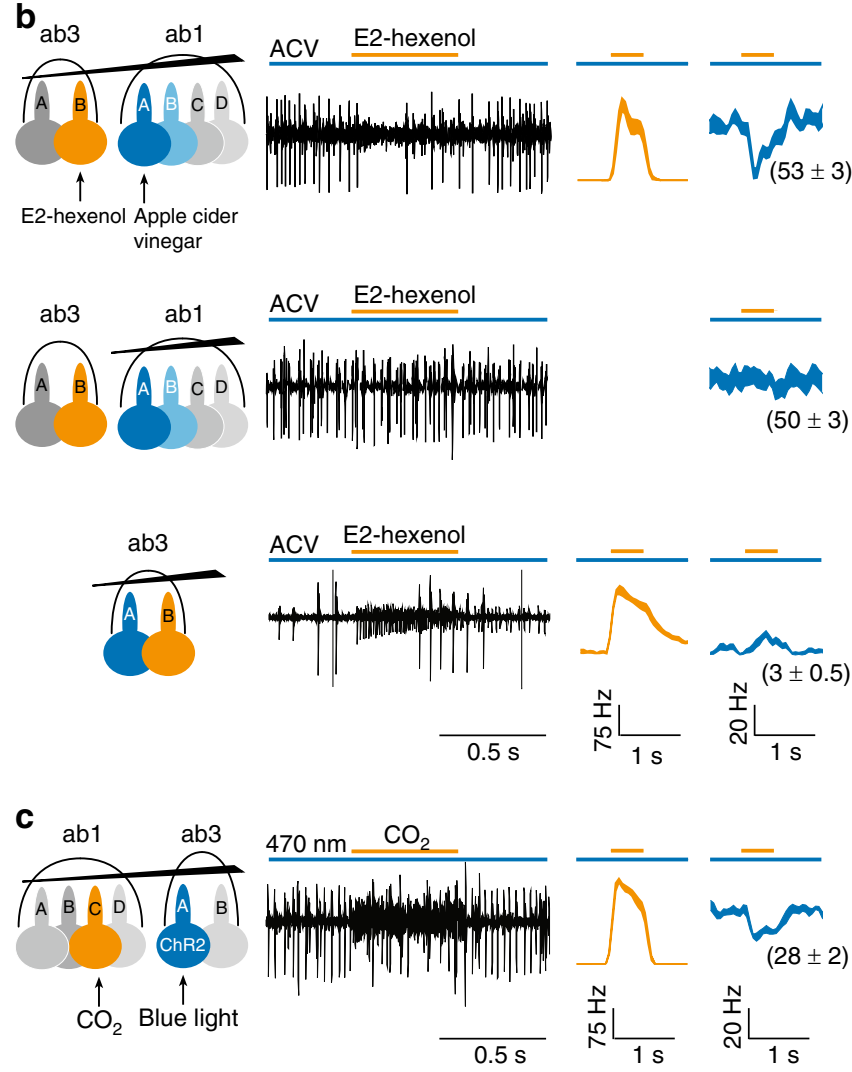

level of both ORNs ${ }^{11}$. To overcome these limitations, we sought to define the strength of ephaptic inhibition by other means.

According to an electric circuit model, neighboring ORNs shared the same electric field, also known as the transepithelial potential, which provides the driving force for odor-induced transduction currents ${ }^{12}$. As such, activation of one ORN reduces the transepithelial potential, thereby shunting currents away from its neighbor to cause ephaptic inhibition ${ }^{11}$. Thus, the degree to which activation of one ORN reduces the transepithelial potential, which can be measured as a change in the local field potential (LFP), indicates the magnitude of its ephaptic influence. The larger the LFP responses of one ORN, the more it can inhibit its neighbor.

To determine the relationship between ephaptic inhibition and LFP responses, we first identified odorants that strongly and selectively activate only one of the grouped ORNs (henceforth referred to as "private odorants", Supplementary Fig. 2 and Supplementary Table 1). Using private odorants for the $a b 2$ ORNs (ab2A: methyl acetate; ab2B: ethyl 3-hydroxy butyrate), we
Fig. 1 Direct electrical interaction drives lateral inhibition between ORNs. a The sustained response of ab1A and ab1B was cross-inhibited by the transient activation of ab1C. Top: ab1A/B responded (large spikes in trace) to a sustained stimulus of apple cider vinegar $\left(3 \times 10^{-3}\right.$ dilution, $\mathrm{v} / \mathrm{v}$ in water, long blue bar). A 500-ms pulse of carbon dioxide $\left(\mathrm{CO}_{2}\right.$, orange bar above trace) activated ab1C (small spikes). The responses of ab1A and ab1B were inhibited by the $\mathrm{CO}_{2}$ stimulus (decreased frequency of large spikes). In the average spike responses on the right, the orange trace represents the response of ab1C to $\mathrm{CO}_{2}$, and the blue trace represents the response of the large-spike neurons. ab1A and ab1B spikes could not be sorted reliably and were grouped. The sustained responses of the large-spike ORNs (blue traces) are indicated in the parentheses (spikes $\mathrm{s}^{-1}$ ). Line width indicates $\mathrm{s}$. e.m. Bottom: in the $\mathrm{CO}_{2}$ receptor mutant flies, $\mathrm{CO}_{2}$ did not activate ab1C or inhibit the sustained response of ab1A/B to vinegar. $\mathbf{b}$ Cross-inhibition between electrically coupled ORNs. Top: a tungsten electrode was used to electrically couple two adjacent sensilla: ab3 (distal) and ab1 (proximal). Activation of ab1A/B by a sustained stimulus of vinegar $\left(3 \times 10^{-3}\right.$ dilution) was inhibited by the excitation of ab3B housed in the electrically coupled sensillum by a pulse of E2-hexenol $\left(10^{-3}\right)$. Middle: when the same two sensilla were no longer electrically coupled, E2-hexenol ceased to inhibit the sustained response of ab1A/B to vinegar. Bottom: when ab3 sensillum was recorded alone, no cross-inhibition was observed between ab3A and ab3B by the same odor stimuli. c $A$ tungsten electrode was used to electrically couple two adjacent sensilla: ab1 (distal) and ab3 (proximal). The sustained ab3A activity in response to optogenetic activation was inhibited by a pulse of $\mathrm{CO}_{2}$, which excited ab1C housed in the electrically coupled sensillum. Light stimulation: $470 \mathrm{~nm}, 2.66 \mu \mathrm{W} \mathrm{mm}{ }^{-2}$. Of note, in the bridged configuration, spike amplitudes of the ORNs housed in the distal sensillum are typically smaller than those in the proximal sensillum. $n=9$ for all recordings. ORN olfactory receptor neuron

recorded LFP responses to 0.5 -s pulses of the private odorants, delivered either as individuals or as concurrent binary odor mixtures (Supplementary Figure 3a). If there is no ephaptic inhibition, the LFP response to a binary odor mixture is expected to be the linear sum of the responses to its constituents. Thus, the difference between the linear sum and the measured LFP response indicates the magnitude of ephaptic inhibition.

As expected, we observed bidirectional inhibition using binary odor mixtures. Concurrent activation of ab2B by ethyl 3-hydroxy butyrate attenuated the LFP responses of ab2A to methyl acetate, and so did activation of ab2A to ab2B responses (Supplementary Fig. 3b). Importantly, the degree of inhibition increased with higher LFP responses (Supplementary Fig. 3c). Henceforth, we measured the LFP responses of an ORN to evaluate its ephaptic influence on its neighbor.

Grouped ORNs have different maximal field responses. To compare the LFP responses, we first focused on the ab2 ORNs, for which multiple private odorants are available (Supplementary Table 1). When ab2A was stimulated by increasing concentrations of methyl acetate, its LFP responses plateaued at $\sim 23 \mathrm{mV}$. In comparison, the near-saturated LFP responses elicited by ethyl 3hydroxy butyrate, a private odorant for ab2B, were markedly smaller, only $\sim 10 \mathrm{mV}$ (Fig. 2a, top panel). Importantly, the LFP amplitudes were characteristics of the ORNs, regardless of the position of the electrode along the sensillum. We note that ethyl 3-hydroxy butyrate activates ab2B strongly and effectively; at $3 \times 10^{-4}$ dilution, the odorant elicited a high spike response in ab2B $\left(\sim 250\right.$ spikes s$\left.^{-1}\right)$, comparable to the spike responses of ab2A to methyl acetate (Supplementary Fig. 4 and Supplementary Table 1). Thus, the difference in the ab2A and ab2B LFP responses is unlikely to have originated from different efficacies of 
a

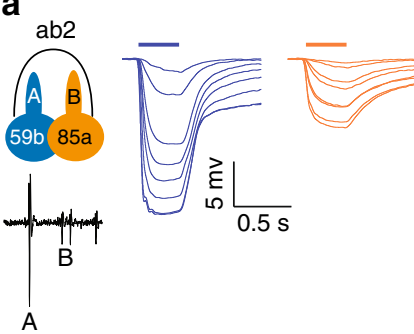

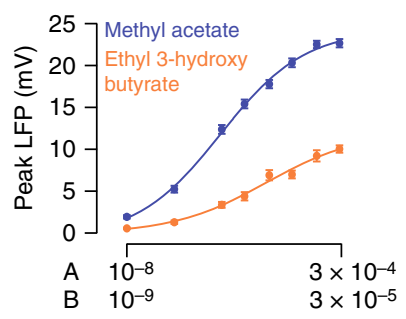

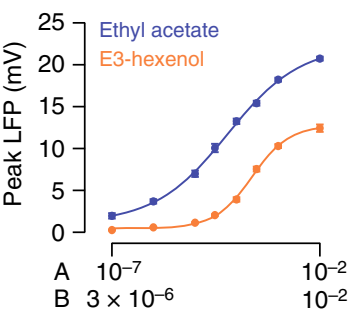

d

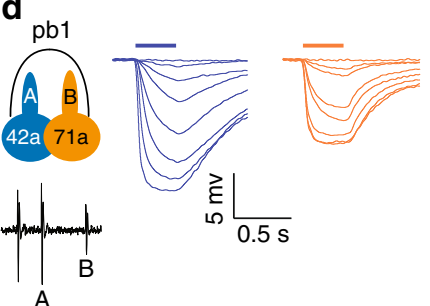

A

$\mathbf{f}$

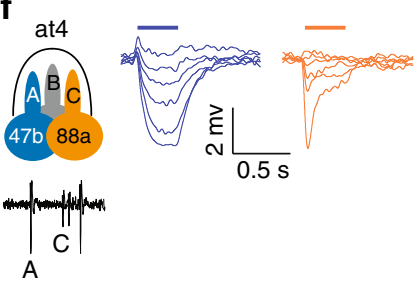

g

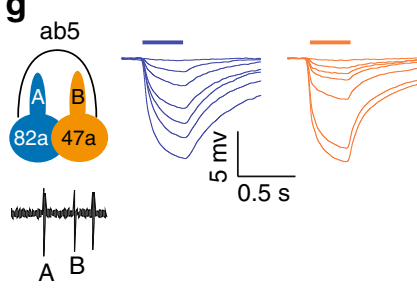

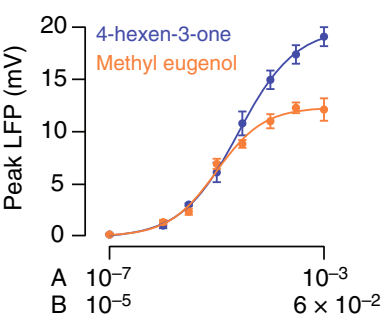
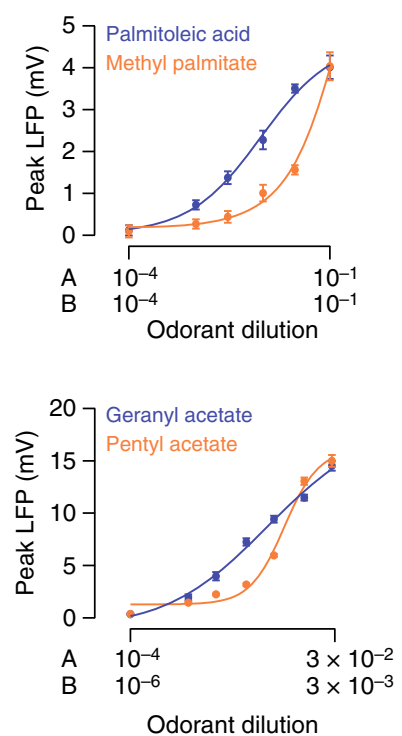

b
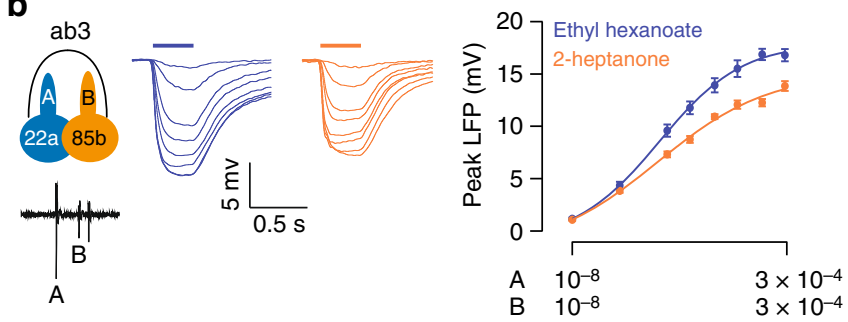

C $\mathrm{ab} 4$
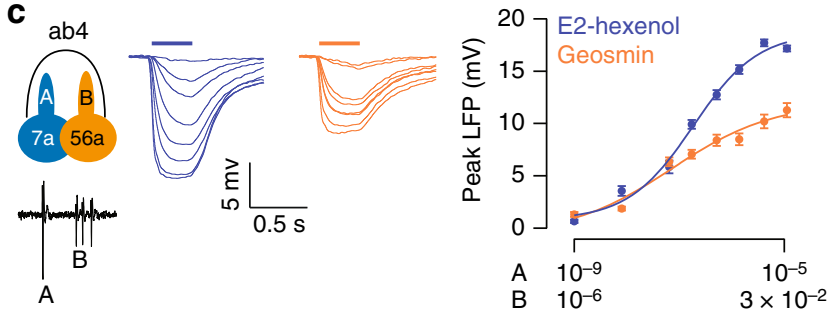

e
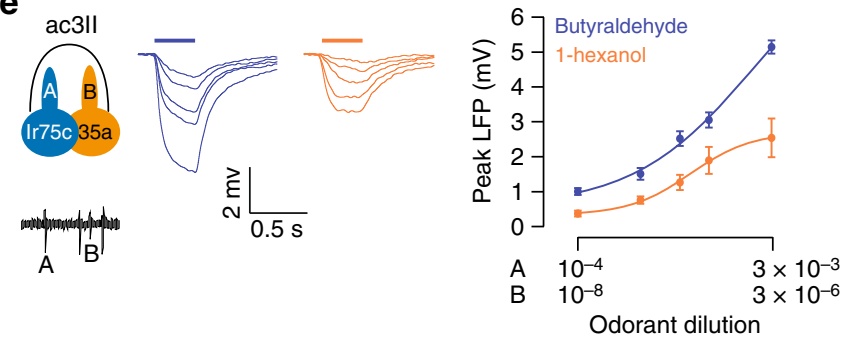

h

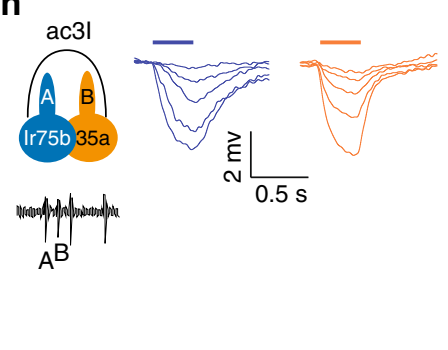

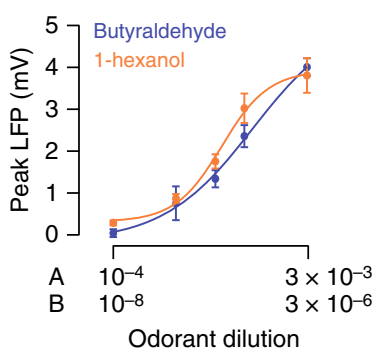

Fig. 2 Comparison of the field responses of grouped neurons. a-f Dose-response relationships of grouped ORNs that have distinct extracellular spike amplitudes. a Left: Spontaneous activity of ab2A (large spike) and ab2B (small spike). Middle: Average local field potential (LFP) responses of ab2A (blue traces) and ab2B (orange traces). Paired ORNs in the same sensilla were recorded in response to their respective private odorants at increasing concentrations. Right: Dose-response relationships of ab2A (blue) and ab2B neurons (orange) to methyl acetate and ethyl 3-hydroxy butyrate (top) or ethyl acetate and E3-hexenol, respectively (bottom). The absolute values of the peak LFP responses are shown. The highest and lowest concentrations of the " $\mathrm{A}$ " and " $\mathrm{B}$ " odorants are indicated logarithmically on the $x$-axis and aligned arbitrarily to facilitate comparison. b-f Additional sensillum types (ab3, ab4, pb1, ac3ll and at4) were examined in a similar manner, as shown in $\mathbf{a} . \mathbf{g}$, $\mathbf{h}$ Average LFP responses and dose-response curves of grouped ORNs that exhibit similar extracellular spike amplitudes ( $\mathbf{g}$ ab5; $\mathbf{h}$ ac3I). $n=9$ pairs of ORNs, except for ac3: $n=6$ pairs, mean \pm s.e.m. Fit is with the Hill equation. Source data are provided as a Source Data file. ORN olfactory receptor neuron

the odorants. To verify this interpretation, we tested another pair of private odorants for the ab2 ORNs (ab2A: ethyl acetate; ab2B: E3-hexenol). We found that the near-saturated LFP response of $\mathrm{ab} 2 \mathrm{~A}$ remained markedly larger than that of ab2B (Fig. 2a, bottom panel). These results indicate that strong activation of $\mathrm{ab} 2 \mathrm{~A}$ can reduce the shared electric field more than that of ab2B. In this context, ab2A is the dominant ORN in the pair.

To test whether other large-spike " $A$ " neurons also exert greater ephaptic influence upon their neighbors, we extended our analysis to additional sensillum types, selected on the basis of 
whether private odorants are available (Supplementary Table 1). In total, our analysis included eight sensilla covering the major sensillum classes in the antenna and maxillary palp: large basiconic (ab2 and ab3), small basiconic (ab4 and ab5), coeloconic (ac3I and ac3II), trichoid (at4), and palp basiconic $(\mathrm{pb} 1)^{14,26,27}$.

In five out of the eight sensillum types examined, grouped ORNs exhibited notably different maximal LFP responses, including ORNs housed in the ab2, ab3, ab4, pb1, and ac3II sensilla. In each case where near-saturated responses were observed, the "A" neuron showed greater maximal LFP responses than its small-spike neighbor (Fig. 2a-e). Analysis of the at 4 ORNs could not be completed because their private odorants failed to elicit near-saturated LFP responses even at a high concentration $\left(10^{-1}\right)$ (Fig. 2f; see Methods for details).

We note that a small minority of Drosophila olfactory sensilla contain ORNs of similar spike amplitudes, such as the ab5 and ac3I sensilla (Supplementary Fig. 5). In both cases, the nearsaturated LFP responses of the grouped ORNs were nearly indistinguishable (Fig. 2g, h), suggesting that these neurons exert comparable ephaptic influence upon each other.

Overall, our results indicate that grouped ORNs exhibiting markedly different spike amplitudes likely exert unequal ephaptic influence upon each other, and most large-spike " $\mathrm{A}$ " neurons are the dominant neurons in this context. The relative spike amplitudes of grouped ORNs thus indicate the rank order of their ephaptic influence. In agreement with this notion, we found that transient activation of ab1C was more effective than ab1D in inhibiting the sustained response of ab1A ORNs (Supplementary Fig. 6).

Grouped ORNs have different tendencies to change spike rates. Next we compared the susceptibility of grouped ORNs to ephaptic influence. Using single-sensillum recordings, we simultaneously recorded the LFP responses, which originate in the sensory cilia and reflect transduction currents ${ }^{28,29}$, as well as the spike responses, which result from depolarization of the ORN soma. We used the spike/LFP ratio to evaluate the degree to which the neuron alters its spiking rate in relation to changes in its LFP response. For an ORN with a higher spike/LFP ratio, the neuron will experience a greater reduction in its spike response when the shared driving force is diminished by the activation of its neighbor. The ORN will therefore be more susceptible to ephaptic influence.

Upon stimulation, the frequency of ORN spiking is determined by depolarization of the soma, which is the product of the transduction current and somatic input resistance (see Methods). Although the transduction current cannot be measured directly in an in vivo preparation, it is thought to give rise to the LFP responses ${ }^{28,30,31}$. Hence, we used the LFP responses as a proxy for transduction currents in the following analysis.

We first stimulated ab3A and ab3B with 0.5-s pulses of their respective private odorants at increasing concentrations (Fig. 3a). By plotting the peak spike rate as a function of the peak LFP amplitude (absolute value), we found that the spike/LFP ratio was significantly higher for ab3B (Fig. 3b), indicating that ab3B is more susceptible to ephaptic influence than ab3A. However, the spiking rate of an insect ORN is determined not only by the amplitude but also by the kinetics of the $\mathrm{LFP}^{28}$. Thus, the variability in LFP kinetics introduced by differing transduction kinetics or odorant dynamics ${ }^{28,32}$ may confound our spike/LFP analysis.

To address this concern, we used an optogenetics approach so that identical stimuli can be used to activate grouped ORNs. When light activated either ab3A or ab3B expressing
Channelrhodopsin2 (H134R-ChR2) ${ }^{33}$, the LFP responses increased with stimulus intensity (Fig. 3c). Consistent with our earlier analysis with odor stimulation, the spike/LFP ratio was also significantly higher for ab3B in the optogenetic assay (Fig. 3d). Of note, a similar spike/LFP relationship was observed even with a lower level of functional H134R-ChR2, achieved by lowering the concentration of retinal fed to flies (Supplementary Fig. 7). Therefore, the difference in the spike/LFP ratios between ab3A and ab3B was unlikely to have been affected by the exact expression level of H134R-ChR2.

We note that direct activation of H134R-ChR2 in the soma could in principle also contribute to ORN spike responses. To evaluate the impact of this possibility, we performed additional control experiments. We ectopically expressed Or83c, an odorant receptor tuned to farnesol ${ }^{34}$, in either ab3A or ab3B to allow for direct comparison of their responses to identical odor stimuli. Farnesol $\left(\leq 2 \times 10^{-2}\right.$ dilutions $)$ did not activate the cognate receptors of the ab3 ORNs (Supplementary Fig. 8a). Analyses of farnesol-induced responses in the Or83c-expressing ab3 ORNs indicated that the spike/LFP ratio was also significantly higher for ab3B (Supplementary Fig. 8b, c). This result suggests that somatic activation of H134R-ChR2, if any, is unlikely to have altered the relative spike/LFP relationship between neighboring ORNs.

We then extended the optogenetic analysis to five additional sensillum types, including ab1, ab2, ab4, pb1, and at4. In each case, the small-spike ORNs exhibited significantly higher spike/ LFP ratios than the neighboring "A" ORNs (Fig. 3e), except for the at4 ORNs of which the spike amplitudes were the least distinct (Supplementary Fig. 5g). Notably, even in the ab1 sensillum that houses four ORNs, the relative spike amplitudes of the neurons $(\mathrm{ab} 1 \mathrm{~A} \geq \mathrm{ab} 1 \mathrm{~B}>\mathrm{ab} 1 \mathrm{C}>\mathrm{ab} 1 \mathrm{D})$ remained indicative of the rank order of the spike/LFP ratios $(\mathrm{ab} 1 \mathrm{~A} \leq \mathrm{ab} 1 \mathrm{~B}<\mathrm{ab} 1 \mathrm{C}<\mathrm{ab} 1 \mathrm{D})$ (Fig. 3e).

To test whether grouped ORNs with similar spike amplitudes have similar spike/LFP ratios, we examined ab5 and ac3I, the two sensilla which house ORNs of similar spike amplitudes (Supplementary Fig. 5h, i). For technical reasons, we could not perform the optogenetic analysis in these sensilla (see Methods for details). Therefore, we ectopically expressed Or83c in either ab5A or ab5B to compare their responses to the same odorant, farnesol. Analyses of farnesol-induced responses indicated that ab5A and ab5B indeed have similar spike/LFP relationships (Fig. 4a, b). Consistent with this result, analyses with private odorants for ab5 and ac3I ORNs also suggested that these neighboring neurons exhibit similar spike/LFP relationships (Fig. 4c-f).

Taken together, our odorant and optogenetic analyses show that in most sensilla, ephaptic interactions are asymmetric in that the large-spike " $\mathrm{A}$ " neuron is dominant. This dominance is due to (1) the " $A$ " neuron's greater ability to reduce the shared driving force (measured as LFP responses, Fig. 2), and (2) its lower susceptibility to ephaptic influence, evaluated by its lower propensity to change spiking rate in response to changes in LFP (Fig. 3).

Overexpression of odorant receptors. What determines the difference in the maximal LFP responses of the "A" and "B" ORNs? One possibility is that the density of odorant receptors expressed in the "B" ORNs is typically lower than in the " $A$ " ORNs. As such, fewer receptors in the "B" neuron can be activated by odor stimulation, resulting in a smaller increase in conductance and subsequently a smaller LFP response. To test this possibility, we overexpressed the cognate receptor, Or85a, together with its obligatory coreceptor $\mathrm{Orco}^{35}$, in the ab2B ORNs. 
a

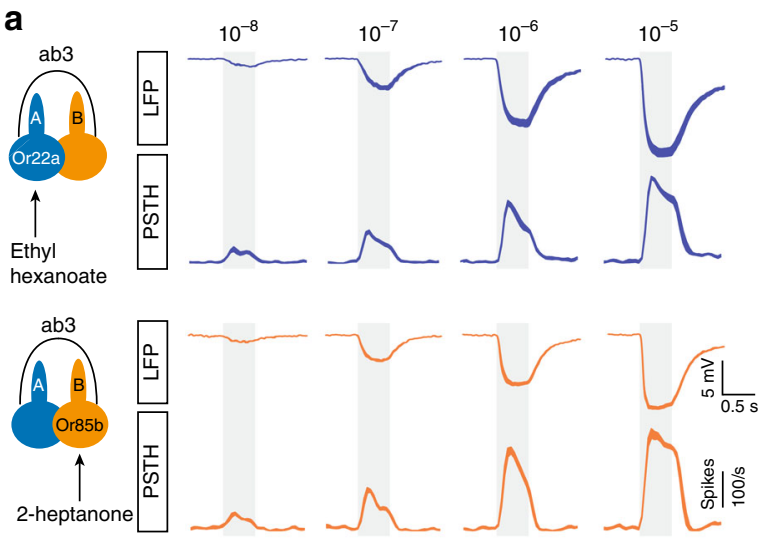

C

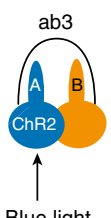

$0.18 \mu \mathrm{W} / \mathrm{mm}^{2} \quad 0.51 \mu \mathrm{W} / \mathrm{mm}^{2} \quad 0.77 \mu \mathrm{W} / \mathrm{mm}^{2} \quad 1.07 \mu \mathrm{W} / \mathrm{mm}^{2}$
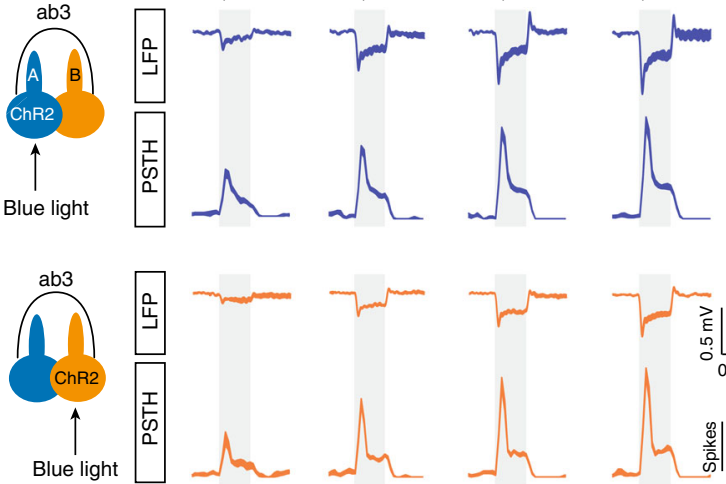

e
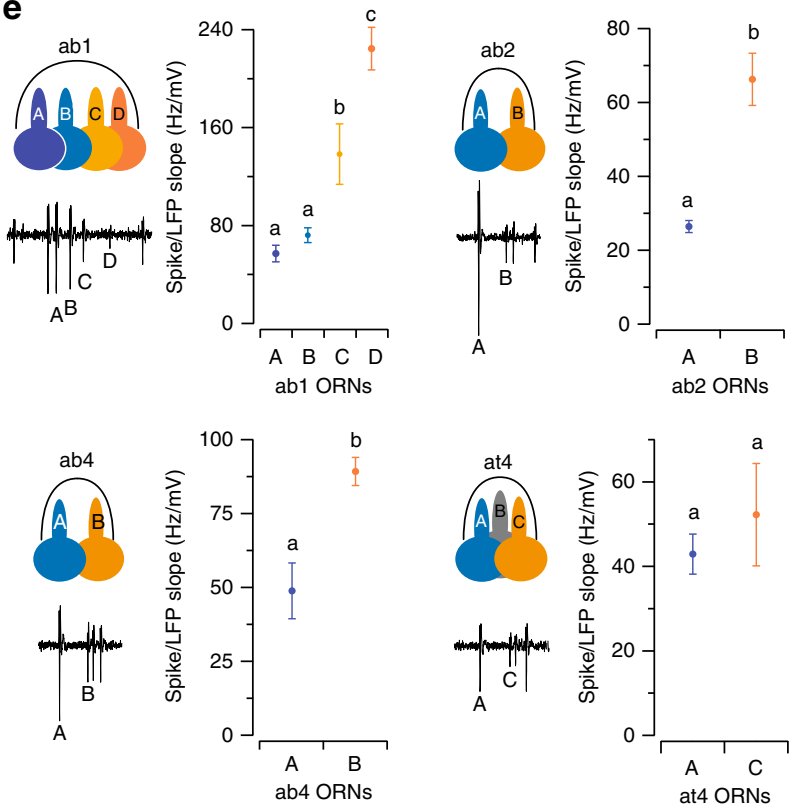

b
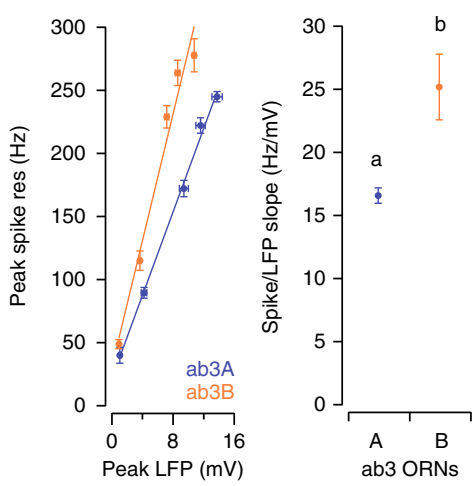

d
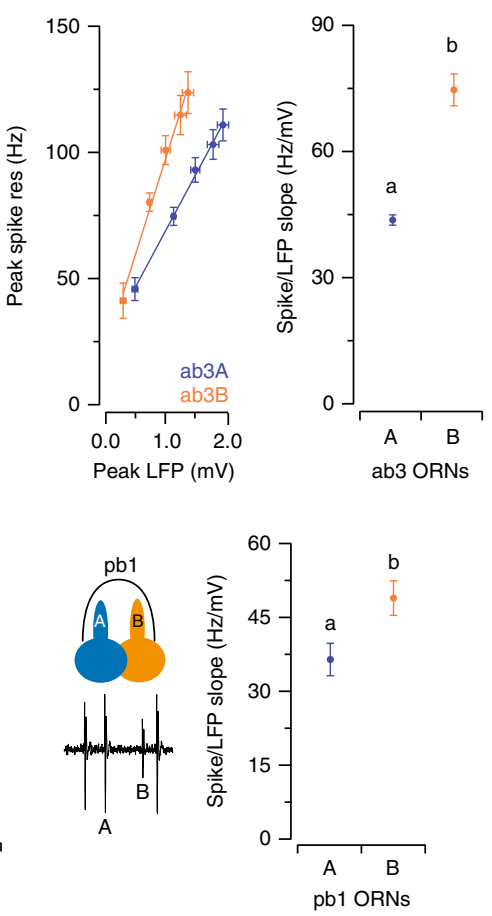

Fig. 3 Comparison of the spiking properties of grouped neurons. a ab3 ORNs were selectively activated by their respective private odorants. a Average LFP responses and the corresponding spike responses are shown for ab3A (blue) and ab3B (orange). b Peak spike responses are plotted as a function of peak LFP responses (left). The absolute values of the peak LFP responses were used for all analyses. Lines indicate linear fits $(y=a x+b) . n=9$ pairs of ORNs, mean \pm s.e.m. The respective " $a$ " coefficients (spike/LFP slope) are plotted for comparison (right). Error bars $=$ s.d. $\mathbf{c}, \mathbf{d}$ Similar to (a, $\mathbf{b})$ except that ab3A and ab3B were activated optogenetically. H134R-Channelrhodopsin2 (ChR2) was expressed in either ab3A or ab3B by the GAL4-UAS system. ORNs were activated by $500-\mathrm{ms}$ pulses of blue light ( $470 \mathrm{~nm}$, irradiances are indicated above). $n=9$, parallel experiments. e Optogenetic analyses in five additional sensilla. ORNs expressing H134R-ChR2 were activated by blue light and the peak spike and LFP responses were analyzed as shown in $(\mathbf{d})$. Error bars $=\mathrm{s} . \mathrm{d}$. $n=9$, parallel experiments. Statistical analysis was performed with ANCOVA and significant differences $(P<0.05)$ are denoted by different letters. The $P$ values are b 0.012; d 0.0001; e ab1A-B: 0.529, ab1A-C: 0.044, ab1A-D: 0.001, ab1B-C: 0.041, ab1B-D: 0.004, ab1C-D: 0.042, ab2A-B: 0.001, pb1A-B: 0.041, ab4A-B: 0.018, at4A-C: 0.566. Source data are provided as a Source Data file. LFP local field potential, ORN olfactory receptor neuron, ANCOVA analysis of covariance 
a
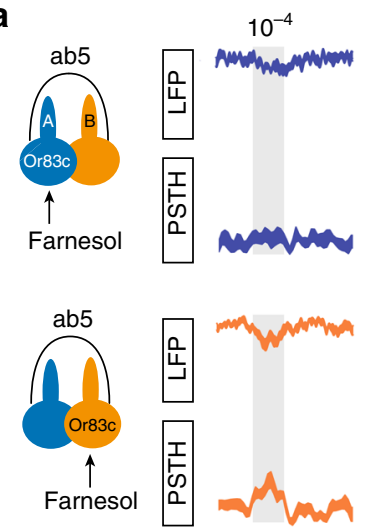

C
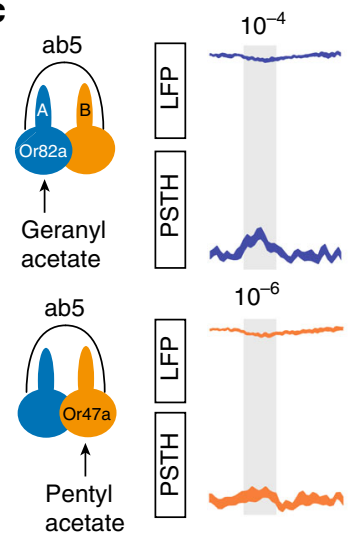

e
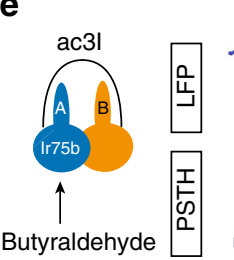

$10^{-8}$
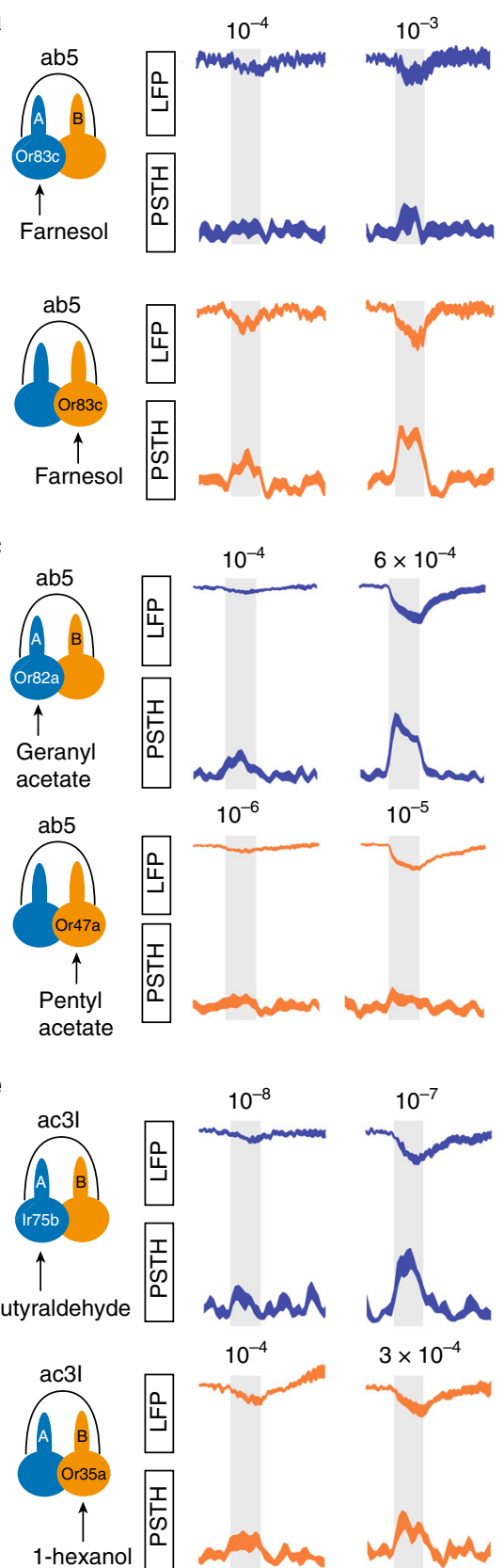

$10^{-5}$

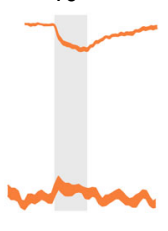

$10^{-4}$
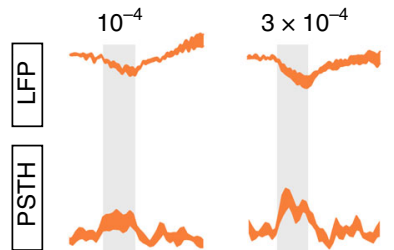
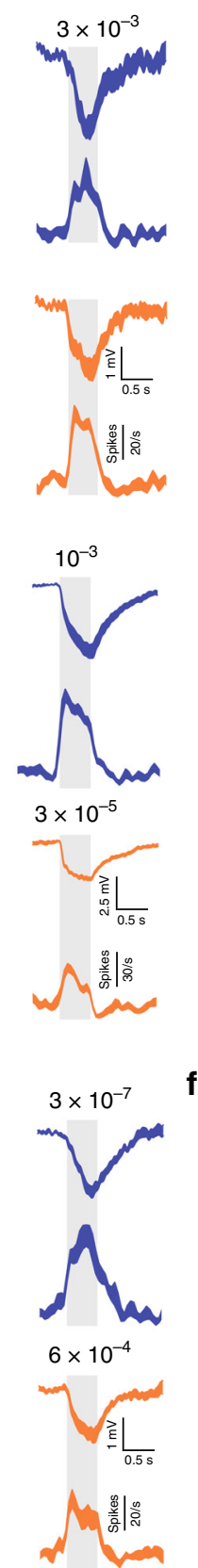

b
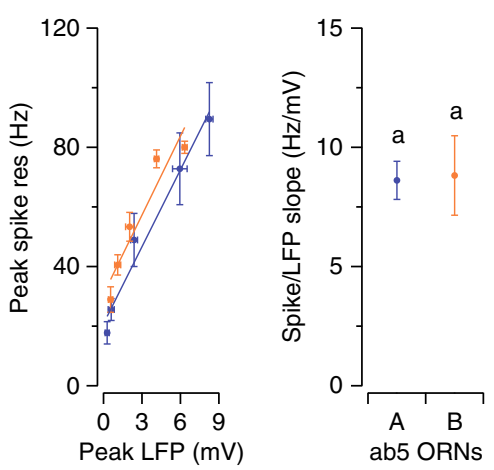

d
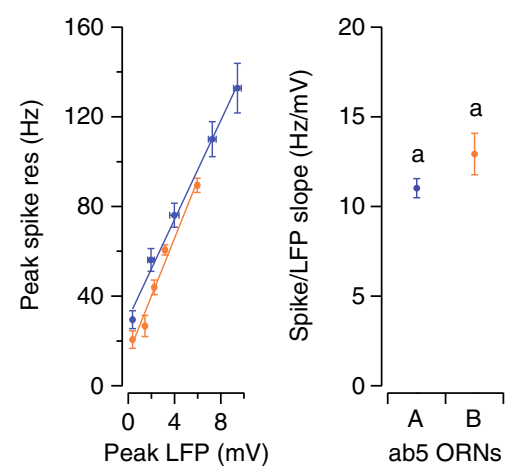

Fig. 4 Spike-LFP analysis in grouped ORNs of similar spike amplitudes. a, b Or83c was ectopically expressed in either ab5A or ab5B using the GAL4-UAS system. ORNs were selectively activated by the odorant, farnesol, at different concentrations. a Average LFP responses and the corresponding spike

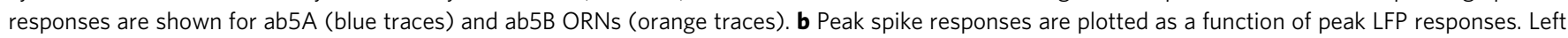
panel: Lines indicate linear fits $(y=a x+b) \cdot n=9$, mean \pm s.e.m. Right panel: The respective " $a$ " coefficients (spike/LFP slope) for ab5A and ab5B are plotted for comparison. Error bars = s.d. c-f Similar to (a, b) except that ab5 ORNs (c, d) or ac3I ORNs (e, f) were selectively activated by their cognate private odorants at different concentrations. $n=9$ pairs of ORNs for (c, d) and six pairs for (e, f), mean \pm s.e.m. Statistical analysis was performed with ANCOVA and significant differences $(P<0.05)$ are denoted by different letters. The $P$ values are b $0.08 ; \mathbf{d} 0.165 ; \mathbf{f} 0.227$. Source data are provided as a Source Data file. LFP local field potential, ORN olfactory receptor neuron, ANCOVA analysis of covariance

Immunostaining of the epitope-tagged odorant receptors indicated successful dendritic targeting (Supplementary Fig. 9).

In the wild-type control, strong activation of ab2A resulted in a large LFP response $(\sim 28 \mathrm{mV})$, markedly higher than the nearsaturated LFP response of ab2B $(\sim 12 \mathrm{mV})$ (Fig. 5a, left panel). Interestingly, in the $\mathrm{ab} 2 \mathrm{~B}$ overexpressing Or85a, the maximal LFP response remained similar to that of the control, well below the ab2A counterpart (Fig. 5a, right panel). That is, overexpression of Or85a did not increase the LFP responses of ab2B. A plausible scenario is that the expression level of endogenous Or85a is already high, likely close to saturation, such that overexpressed Or85a only replaces the endogenous receptor in the sensory dendrite without further increasing its receptor density. Thus, the smaller LFP response of a "B" ORN is unlikely to have arisen from a lower receptor density.

Swapping odorant receptors does not alter the LFP response. Next, we examined the contribution of receptor identity to the maximal LFP responses. In a sensillum, neighboring ORNs 
a
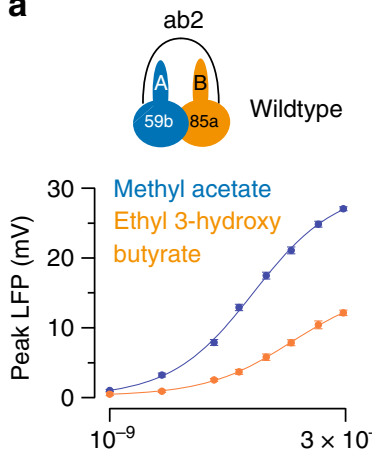

Odorant dilution
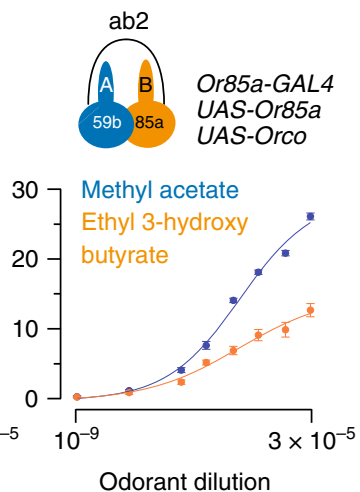

b
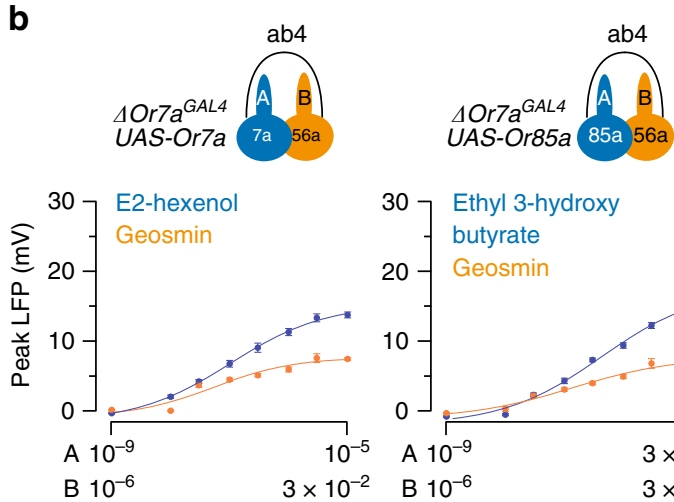

Odorant dilution

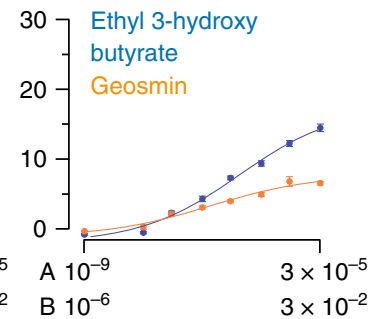

Odorant dilution

Fig. 5 Overexpressing or swapping odorant receptors does not change the maximal LFP responses of an ORN. a Receptor overexpression in the ab2B ORNs. ab2A responded to methyl acetate and ab2B to ethyl 3-hydroxy butyrate. Left panel: in control flies, ab2A had a greater near-saturated LFP response than ab2B (similar results observed in Fig. 2a). Right panel: overexpression of the odorant receptor complex, Or85a/Orco, in ab2B did not increase its maximal LFP responses. b Receptor swap in the ab4A ORNs. Left panel: genetic rescue of Or7a expression in the $\triangle O r 7 a G A L 4$ mutant flies ( $\triangle a b 4 A: O r 7 a)$ restored the responses to E2-hexenol. The neighboring neuron, ab4B, responded to geosmin. The rescued ab4A ORNs had a greater near-saturated LFP response than $\mathrm{ab} 4 \mathrm{~B}$, as in wild-type flies. The highest and lowest concentrations of the " $\mathrm{A}$ " and " $\mathrm{B}$ " odorants are indicated logarithmically on the $x$-axis and aligned arbitrarily to facilitate comparison. Right panel: ectopic expression of Or85a, the cognate receptor of ab2B, in $\Delta$ ab4A resulted in a similar nearsaturated LFP response as in $\triangle$ ab4A:Or7a. $n=9$ pairs of ORNs, mean \pm s.e.m. All results in $\mathbf{a}$ or $\mathbf{b}$ are from parallel experiments. Source data are provided as a Source Data file. LFP local field potential, ORN olfactory receptor neuron

express different odorant receptors ${ }^{17,18,36}$. Given that Drosophila odorant receptors are ligand-gated cation channels ${ }^{37-39}$, it is possible that the conductance of the receptors expressed in the "A" ORNs is typically larger than in the "B" neurons. If so, when the cognate receptor of an " $A$ " ORN is replaced by a " $B$ " neuron receptor, one would expect a marked reduction in the maximal LFP response of the neuron.

To test this possibility, we performed receptor-swap experiments in ab4A, of which the cognate receptor is Or7a $\mathrm{a}^{16}$. We chose ab4A because Or7a receptor mutants $\left(\triangle O r 7 a^{G A L 4}\right)$ are readily available ${ }^{40}$, and because its small-spike neighbor, ab4B, is narrowly tuned to geosmin ${ }^{41}$. In $\triangle O r 7 a^{G A L 4}$ mutants, the response of ab4A $(\triangle \mathrm{ab} 4 \mathrm{~A})$ to an Or7a ligand, E2-hexenol, is completely abolished. Genetic rescue of Or7a in $\triangle \mathrm{ab} 4 \mathrm{~A}(\Delta \mathrm{ab} 4 \mathrm{~A}$ : Or7a) restored the response; the restored LFP dosage curve was similar to that of the wild-type ab4A (Figs. $5 \mathrm{~b}$ and $2 \mathrm{c}$ ). When a "B" neuron receptor (Or85a/ab2B) was expressed in ab4A instead $(\triangle \mathrm{ab} 4 \mathrm{~A}: O r 85 \mathrm{a})$, the LFP dosage curve to an Or85a ligand, ethyl 3hydroxy butyrate (Fig. 5b, left panel), was remarkably similar to that of $\triangle \mathrm{ab} 4 \mathrm{~A}$ :Or7a to E2-hexenol (Fig. 5b, right panel). These results suggest that the characteristic near-saturated LFP responses of an ORN are not influenced by receptor density or identity but likely by other ORN features.

Morphometric analysis of grouped ORNs. What then underlies the asymmetry in ephaptic interactions? The size difference between grouped ORNs is likely a key. In most sensilla, grouped ORNs have differing sizes ${ }^{42}$, and the large-spike " $A$ " neurons were postulated to have larger dendritic calibers ${ }^{43-45}$. However, without a genetically encoded EM marker, it was impossible to assign ORN identity. Notably, the ORN with a larger soma would have a smaller input resistance, which could account for the smaller spike/LFP ratio of the "A" neurons. In addition, a larger surface area of the sensory dendrites could give rise to a larger maximal LFP response, also characteristic of the " $\mathrm{A}$ " neurons. Therefore, we hypothesized that the dominant " $A$ " neuron is larger than its neighbor.

To test this hypothesis, we endeavored to measure the morphometric features of genetically identified ORNs using electron microscopy (EM). To this end, we developed a method termed CryoChem, which allows for optimal morphological preservation of genetically labeled structures for EM imaging ${ }^{46}$. With OR-specific drivers, we expressed an EM marker, APEX2 (enhanced ascorbate peroxidase 2$)^{47}$, in select ORNs to render them electron dense through diaminobenzidine (DAB) labeling. As a proof of principle, we first generated $3 \mathrm{D}$ volumes of at $4 \mathrm{~A}$ $($ Or47b $>$ APEX2) and at4C (Or88a $>$ APEX2) using SBEM (Supplementary Fig. 10a, b). Among the three at4 ORNs, we identified at $4 \mathrm{~A}$ and at $4 \mathrm{C}$ as the largest and intermediate-sized neurons in the group, respectively, and used this information to assign neuronal identity to the unlabeled ORNs. By comparing the APEX2-labeled and unlabeled ORNs, we verified that APEX2/ DAB labeling does not significantly alter the morphometric features of ORNs (Supplementary Fig. 10c).

Dominant " $A$ " ORNs are physically larger than their neighbors. Next, we expanded our morphometric analysis to a total of five sensillum types (ab3, ab4, ac3II, at4, and ab5). In the sensilla where grouped ORNs exhibit distinct spike amplitudes (ab3, ab4, ac3II, and at4), the "A" ORNs were significantly larger than their small-spike neighbors with respect to the soma, inner and outer (sensory) dendrites (Fig. 6a-d, Supplementary Table 2; the inner and outer dendrites are defined based on the location of cilium base). Notably, ephaptic interactions were also asymmetric in these sensilla (with the exception of at 4 where the dose-response analysis was incomplete), supporting our hypothesis that morphometric disparity between grouped ORNs underlies their asymmetrical ephaptic interactions. To further test this hypothesis, we examined the ab5 ORNs, which exert equal ephaptic influence onto each other (Figs. 2 and 4 ) and would thus be predicted to be of similar size. Indeed, in ab5A and ab5B we found that their somata, and likely also the sensory dendrites, were similar in size (Fig. 6e, Supplementary Table 2, see below for disparity in inner dendritic volumes).

Beyond morphometric measurements, our SBEM experiments also revealed novel ORN morphological features. For example, in the basiconic sensilla, but not in other sensillum types, we noticed a marked enlargement of the inner dendrite in every "A" ORN (Fig. 6f). This feature explains why the inner dendrite of ab5A is significantly larger than that of ab5B, despite their otherwise 
a
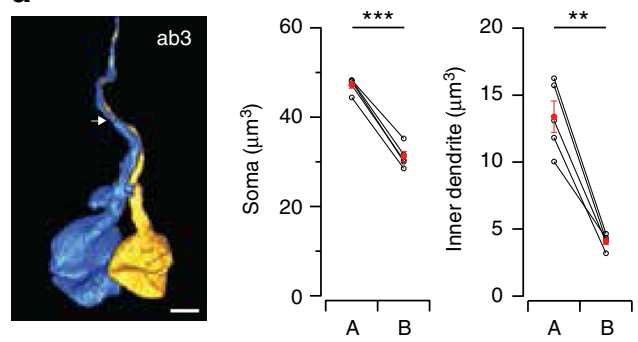

b
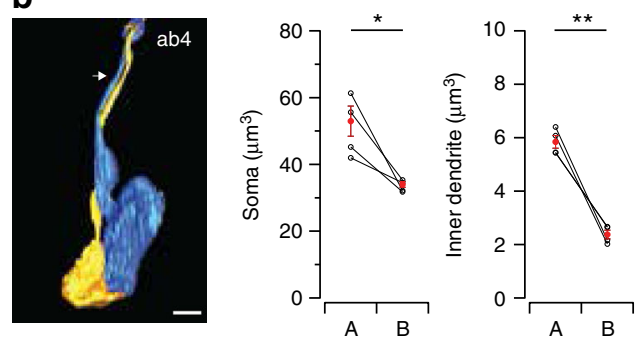

C
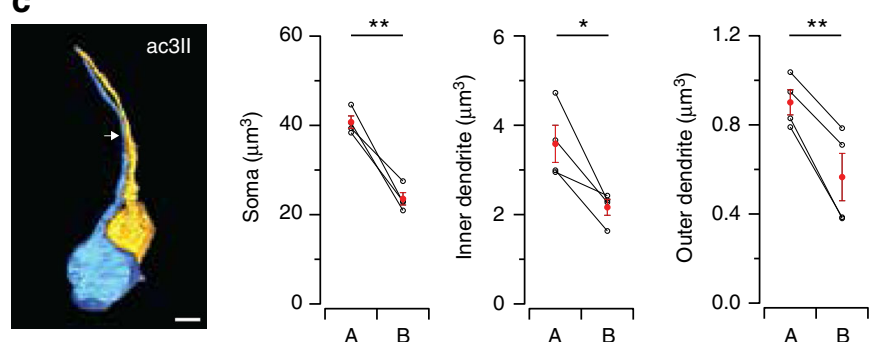

d
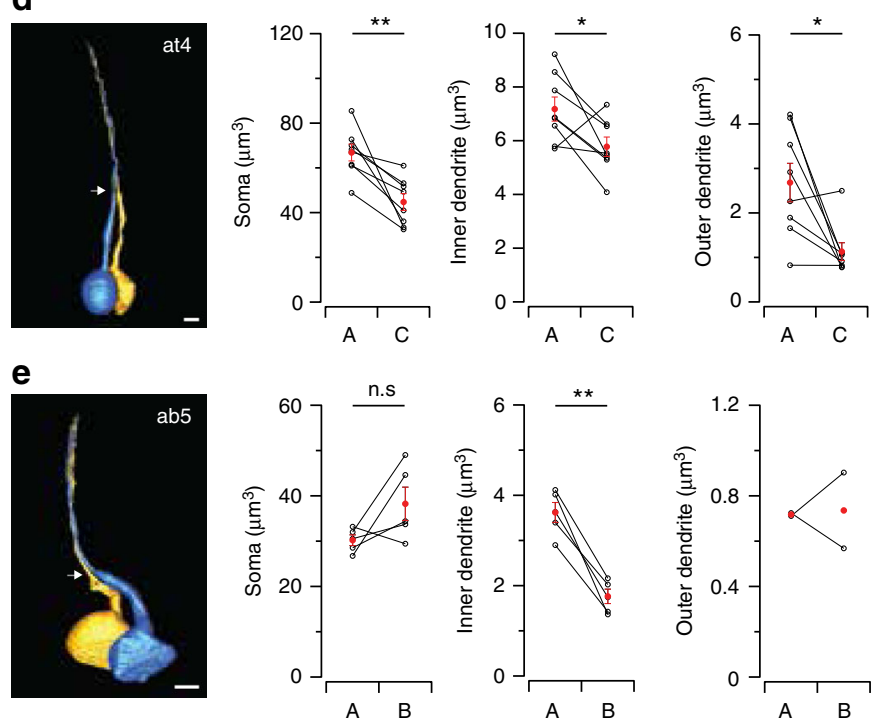
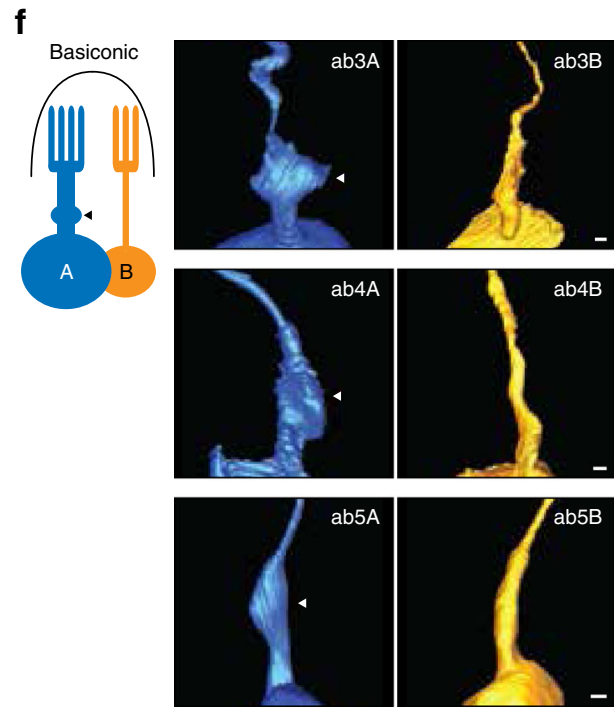

$\mathbf{g}$
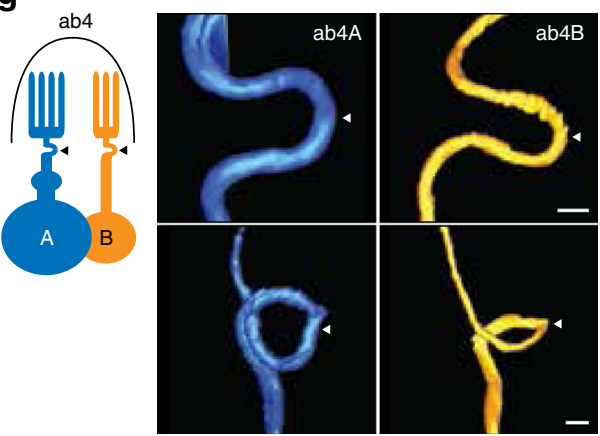

Fig. 6 Systematic morphometric analysis of grouped ORNs. a-e Volumes of the soma, inner and outer dendrites of the paired ORNs in five sensillum types. (Left) Sample 3D reconstruction based on SBEM images. Arrows indicate the cilium base, a constricted region separating the inner and outer dendrites. Due to technical limitations, the outer dendrites of most basiconic sensilla could not be completely reconstructed (see Methods for details). Scale bars: 2 $\mu \mathrm{m}$. Lines connect measurements from paired ORNs, mean \pm s.e.m. $n=4-5$ for all except for at $4, n=8$. ${ }^{\star} P<0.05$, ${ }^{\star \star} P<0.01,{ }^{\star \star \star} P<0.001$, paired $t$ test. The $P$ values are a 0.00004 and $0.001 ; \mathbf{b} 0.033$ and $0.003 ; \mathbf{c} 0.0004,0.035$, and 0.008 ; d $0.004,0.041$, and 0.017 ; e 0.125 and 0.001 . Error bars and statistics are unavailable for the ab5 outer dendrites as only two data points were obtained. $\mathbf{f}, \mathbf{g}$ Novel morphological features. $\mathbf{f}$ An enlargement of the inner dendrite (arrow head) was observed in the "A" ORNs in all three characterized basiconic sensilla. $\mathbf{g}$ A bend or loop (arrow head) was observed in the unbranched outer dendrite of the ab4 ORNs. Scale bar: $500 \mathrm{~nm}$. Source data are provided as a Source Data file. ORN olfactory receptor neuron, SBEM serial block-face scanning electron microscopy 
similar sizes (Fig. 6e). In addition, in the ab4 sensilla, we observed bending or looping of the unbranched outer dendrite around the base of the sensillum (Fig. 6g). These observations further highlight the diversity of ORN morphology among different sensillum types.

An electric circuit model predicts asymmetric interactions. We used mathematical modeling to explain how the morphometric disparity between ORNs contributes to their asymmetric ephaptic interactions. The existing electric circuit model of a sensillum assumes that neighboring ORNs have identical passive electrotonic properties ${ }^{12}$. However, our morphometric analysis suggests otherwise. We therefore took into account that the input resistance of an ORN is inversely proportional to the surface area of the soma, and that the conductance change upon odor stimulation scales with the surface area of the sensory dendrite (Fig. 7a, see Methods for details).

In our circuit model, the ORN-specific parameters are the odorant sensitivity and the surface areas of soma and sensory dendrite. For other modeling parameters, such as the resting membrane potential and membrane resistivity, identical values were assumed for all ORNs. First we evaluated our model by fitting the peak LFP responses of the ORNs housed in the ab3, ab4 and ab5 sensilla, of which electrophysiology and morphometric data were both available. We fitted the responses from the three ORN pairs simultaneously with identical free parameters using the Data2dynamics framework with a nonlinear leastsquares optimization algorithm ${ }^{48}$. The fitted dose-response curves indicated that our circuit model well describes the experimental data (Fig. 7b). They also confirmed that the larger sizes of the "A" neurons result in higher maximal LFP responses, as evidenced in the fittings of the ab3 and ab4 ORNs, which are of different sizes, but not the ab5 ORNs, which are of similar sizes (Fig. 7b). For the ab3 and ab4 sensilla, where measurements for the sensory dendrites were unavailable, the fitted parameters indicate that the dendritic surface areas of $a b 3 \mathrm{~A}$ and $\mathrm{ab} 4 \mathrm{~A}$ are both larger than those of their respective neighbors (see Methods). The model also predicts the relationship between ORN depolarization $\left(\Delta V_{\mathrm{m}}\right)$ and LFP responses (Fig. 7c). Given that the peak spike response of an ORN is a function of $\Delta V_{\mathrm{m}}{ }^{28}$, the smaller $\Delta V_{\mathrm{m}} / \mathrm{LFP}$ slopes of the " $\mathrm{A}$ " neurons are consistent with their smaller spike/LFP ratios (Fig. 3).

Our experimental data indicate that ephaptic interaction is asymmetric between grouped ORNs in most sensilla (Figs. 2, 3). To test whether our model describes asymmetric inhibition, we simulated the transmembrane potential of ab4 ORNs when either $\mathrm{ab} 4 \mathrm{~A}$ or $\mathrm{ab} 4 \mathrm{~B}$ was activated by a private odorant at increasing concentrations. According to our model, depolarization of one ORN hyperpolarizes its neighbor (Eqs. 11 and 12, Methods). We observed this relationship when both ORNs were assumed to be initially inactive (Fig. $7 \mathrm{~d}$, e, $0 \%$ activation) and when one ORN was assumed to be chronically activated by a background odorant, a paradigm used in Fig. 1 and in our earlier work ${ }^{11}$ (Fig. 7 d, e, 50\% activation). We then evaluated the strength of ephaptic inhibition between neighboring ORNs by comparing the degree to which depolarization of one ORN hyperpolarizes its neighbor. A higher value of the ratio (hyperpolarization/ depolarization) indicates stronger ephaptic inhibition. By comparing the absolute values of $\Delta V_{\mathrm{mB}} / \Delta V_{\mathrm{mA}}$ (Fig. $7 \mathrm{~d}$ ) and $\Delta V_{\mathrm{mA}} /$ $\Delta V_{\mathrm{mB}}$ (Fig. 7e), we found that activation of ab4A was indeed more effective in inhibiting $\mathrm{ab} 4 \mathrm{~B}$ than the reverse situation, indicating that A-to-B ephaptic inhibition is stronger than B-to-A inhibition.

In our model focusing on the basiconic ORNs, the only parameters that are different between grouped ORNs are the neuronal size and odorant sensitivity, both of which can in principle contribute to asymmetric ephaptic interaction. To illustrate the impact of neuronal size, we simulated ab4 ORN responses in a hypothetical situation where both ORNs express the same odorant receptor, Or56a. Even with identical odorant sensitivity, the larger size of ab4A was sufficient to yield a larger maximal LFP response (Fig. $7 \mathrm{f}$, top panel) and a smaller increase in the transmembrane potential (Fig. $7 \mathrm{f}$, bottom panel). In other words, morphometric differences alone can account for the asymmetric ephaptic interactions between grouped ORNs.

\section{Discussion}

We demonstrate here that ephaptic interaction alone is sufficient to influence circuit function by driving lateral inhibition between compartmentalized ORNs. What benefits might this form of direct neuronal interaction provide for olfactory coding? Ephaptic interaction between ORNs shapes timing of spiking in ORNs ${ }^{11}$, thereby allowing for a more elaborately patterned neural code in higher processing centers ${ }^{49}$. In addition, the rapid kinetics afforded by ephaptic interaction likely permits fast processing of odor-mixture information in the pheriphery ${ }^{11}$.

Surprisingly, we find that across sensillum types, most largespike " $A$ " neurons can exert greater ephaptic influence onto their neighbors and are also less susceptible to ephaptic influence. Mechanistically, the functional disparity arises from morphometric differences between compartmentalized ORNs. Together, our study describes a highly specialized inhibitory interaction that governs information processing at the earliest stages of olfactory coding. It also establishes the peripheral olfactory system of Drosophila as a model to understand the impact of electric field effects on neural circuit function.

Ephaptic interactions are asymmetric between grouped ORNs with distinct spike amplitudes. Conversely, grouped ORNs exhibiting similar spike amplitudes exert similar ephaptic influence on each other (Figs. $2 \mathrm{~g}, \mathrm{~h}$ and 4 ). This relationship suggests that the extracellular spike amplitude of an ORN and its ephaptic influence are regulated by a common factor. Indeed, as with ephaptic influence, the extracellular spike amplitude of a neuron also negatively correlates with its input resistance ${ }^{50}$. Thus, the larger ORN in a pair, which has the smaller input resistance, is expected to have larger spike amplitude as well as ephaptic dominance over its neighbor.

In addition to fly ORNs, there are reports of asymmetric inhibition between other primary sensory neurons. For example, in fly vision, R7 and R8 photoreceptors of the same ommatidium inhibit each other synaptically. Interestingly, the mutual inhibition between R7 and R8 is also asymmetric ${ }^{51}$. The asymmetry may have arisen from the unequal numbers of reciprocal synapses $^{52}$. In the bumblebee galea sensilla, gap junctionmediated inhibitory coupling is unequal between compartmentalized gustatory receptor neurons ${ }^{53}$. Therefore, although the precise mechanisms may vary, asymmetric lateral inhibition between adjacent primary sensory neurons may represent a conserved computational motif whereby sensory inputs are unequally processed at the periphery before being transmitted to higher brain centers.

Ephaptic interactions between ORNs allow odor-mixture information to be processed by the first neurons of an olfactory circuit $^{11}$. To evaluate the extent to which natural odors activate grouped ORNs simultaneously, we surveyed volatile compounds from several food sources for fruitflies (Supplementary Data 1). From 16 fruits and fermented foods, we identified 51 odorants that can excite at least one ORN with responses $\geq 50$ spike $\mathrm{s}^{-1}$, based on published datasets ${ }^{15,21,41}$. Among them, 30 individual odorants were capable of coactivating neighboring ORNs. For 
a

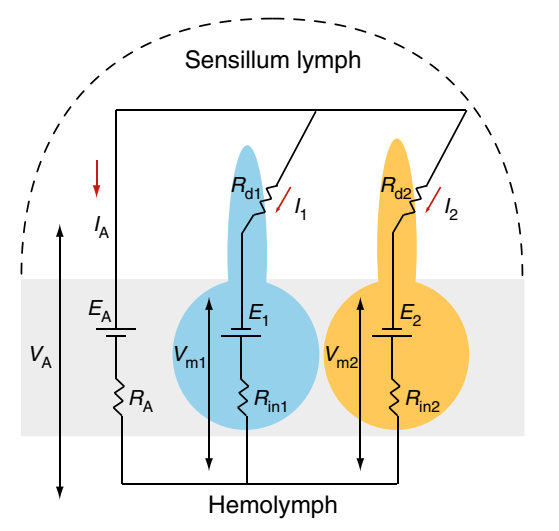

$E_{\mathrm{A}} \quad$ Battery simulating the potential generated by the auxiliary cells

$R_{\mathrm{A}} \quad$ Input resistance of the auxiliary cells

$I_{A} \quad$ Current through the auxiliary cells

$E_{1} \& E_{2} \quad$ Battery simulating the transmembrane ionic gradients across

the soma of the ORNs

$R_{\text {in1 }} \& R_{\text {in2 }} \quad$ Input resistance of the soma of the ORNs

$R_{\mathrm{d} 1} \& R_{\mathrm{d} 2} \quad$ Input resistances of the sensory dendrites, which are odorant

stimulation dependent

$V_{\mathrm{A}} \quad$ Transepithelium potential

$V_{\mathrm{m} 1} \& V_{\mathrm{m} 2} \quad$ Transmembrane potentials of the ORNs

$I_{1} \& I_{2} \quad$ Current through ORNs

Changes in $V_{\mathrm{A}}$ due to odorant stimulation of ORN1 or 2 are measured as changes in LFP.

b
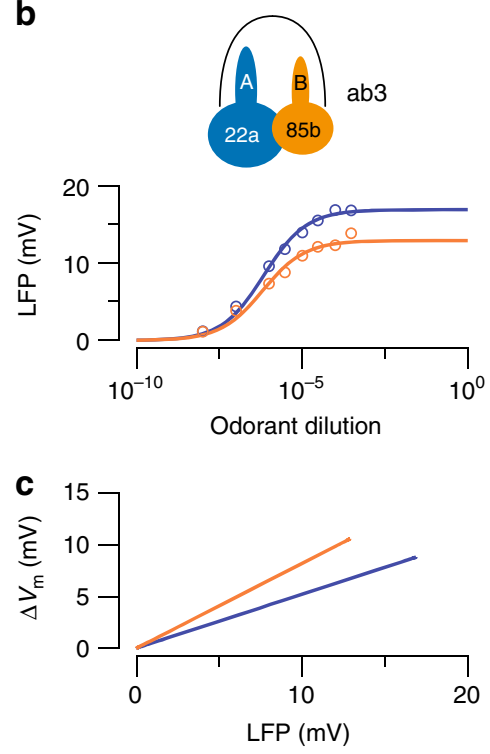

d

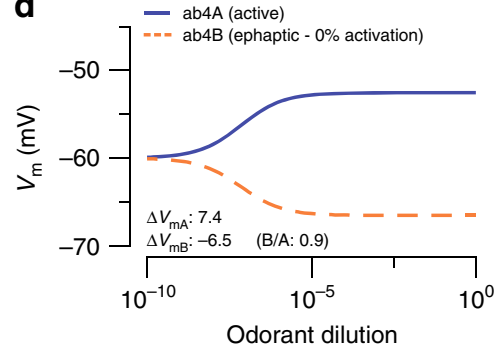

e

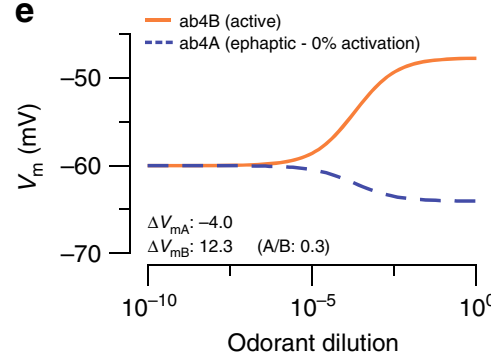

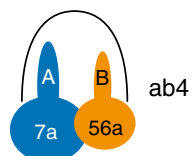
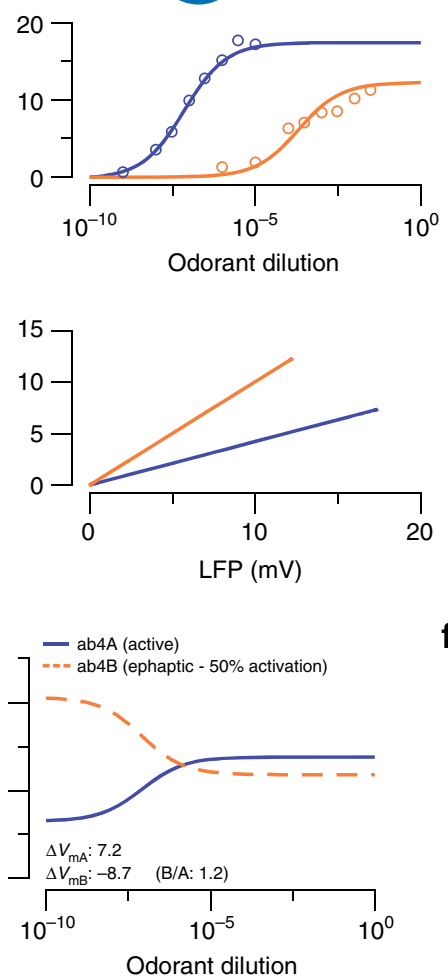

Odorant dilution

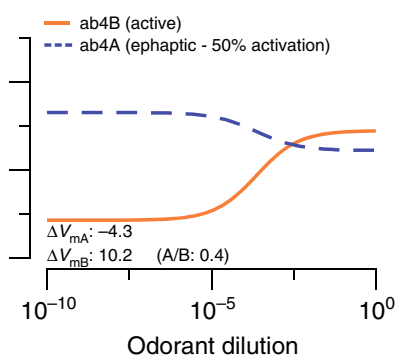

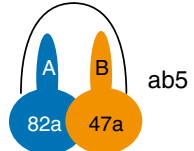
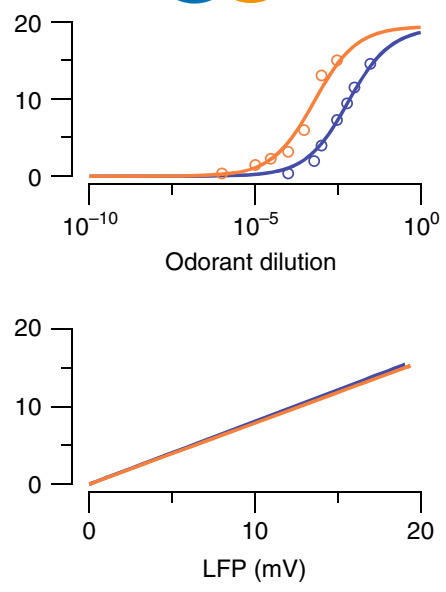

f
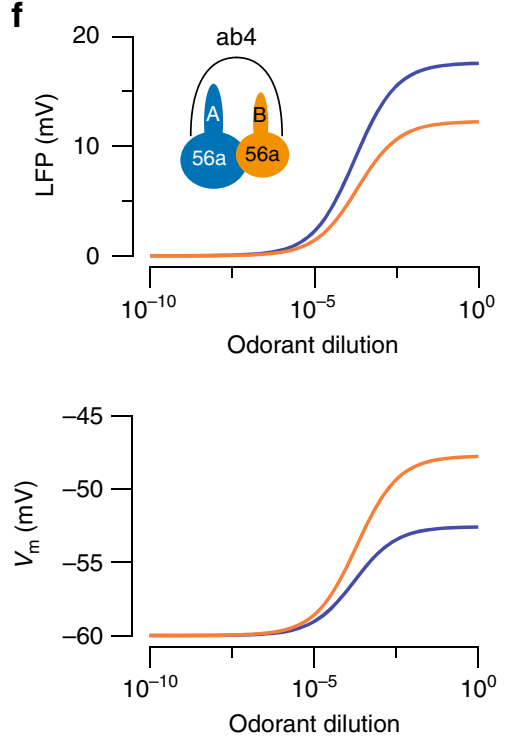

instance, ethyl hexanoate elicited significant responses in both ab3A and ab3B ORNs (Supplementary Data 1). Strikingly, all of the analyzed odor sources contained volatiles that excited at least one pair of the grouped ORNs, suggesting that grouped fly ORNs are commonly coactivated by natural odors.
What then is the significance of asymmetric lateral inhibition between grouped ORNs? In such a situation, this operation may provide a peripheral mechanism for evaluating countervailing signals and favoring the propagation of the input carried by the large-spike ORNs. In a mixture, odorants that excite the small- 
Fig. 7 An electric circuit model for compartmentalized ORNs. a Passive electric circuit model of a sensillum consisting of two ORNs and an auxiliary cell (gray rectangle). b Simultaneous fitting of the LFP responses of the ORNs housed in the ab3, ab4 and ab5 sensilla. Identical parameters were used for all ORNs, except for the morphometric parameters and odorant sensitivity, which are ORN-specific and determined based on the experimental data whenever available. Each curve describes the dose-response relationship of an ORN when activated by a private odorant. Empty circles indicate measured LFP responses. Odorant concentrations are plotted logarithmically on the $x$-axis. $\mathbf{c}$ Simulation of ORN depolarization $\left(\Delta V_{m}\right)$ in relation to the LFP response. $\mathbf{d}$, $\mathbf{e}$ Simulation of the transmembrane potential of the ab4 ORNs $\left(V_{m}\right)$ when either ab4A $(\mathbf{d})$ or ab4B (e) was activated by increasing concentrations of private odorants. Two different activation states of the neighboring ORN ( 0 and 50\%) were considered. Depolarization of one ORN ("active", solid line) ephaptically hyperpolarizes its neighbor ("ephaptic", dashed line). $\Delta V_{m A}$ and $\Delta V_{m B}$ : maximal change in $V_{m}(m V)$ for ab4A and ab4B, respectively. A/B or $\mathrm{B} / \mathrm{A}$ : the absolute value of $\Delta V_{\mathrm{mA}} / \Delta V_{\mathrm{mB}}(\mathrm{A} / \mathrm{B})$ or $\Delta V_{\mathrm{mB}} / \Delta V_{\mathrm{mA}}(\mathrm{B} / \mathrm{A})$. $\mathbf{f}$ Simulation of the LFP responses and transmembrane potential changes of the ab4 ORNs, assuming that both ORNs express the same odorant receptor, Or56a. Except for ab4A odorant sensitivity, all fitting parameters are as in $\mathbf{b}$ for ab4. Blue: large-spike "A" ORN. Orange: small-spike "B" ORN. See Methods for details on modeling, data fitting and parameters. Source data are provided as a Source Data file. ORN olfactory receptor neuron, LFP local field potential

spike ORNs are more likely to be masked by odorants activating their large-spike neighbors. In support of this idea, we previously found that this coupling effect is powerful enough to influence animal behavior and that activation of ab1A by vinegar odors attenuated the aversiveness of $\mathrm{CO}_{2}$ detected by ab1C 11 . In future studies, it will be important to determine how odorants that excite the large-spike ORNs qualitatively differ from those that excite the small-spike neurons.

\section{Methods}

Drosophila stocks. Flies were raised on standard cornmeal medium at $25^{\circ} \mathrm{C}, \sim 60 \%$ relative humidity in an incubator with a 12-h light/dark cycle. Female CS flies 5-7 days post eclosion were used in all experiments unless noted otherwise. For the ablation (UAS-rpr) and optogenetic (UAS-H134R-ChR2) experiments, 5-day-old females were used; for the SBEM (UAS-APEX2) experiments, 6-8-day-old females were used. For further information on genotypes, refer to Supplementary Table 4.

Single-sensillum recordings. A fly was wedged into the narrow end of a truncated plastic $200-\mu$ l pipette tip to expose the antenna, which was subsequently stabilized between a tapered glass microcapillary tube and a coverslip covered with doublesided type ${ }^{54}$. Single-unit recordings were performed as follows. Briefly, electrical activity of the ORNs was recorded extracellularly by placing a sharp electrode filled with $0.6 \times$ sensillum lymph Ringer solution ${ }^{31}$ into a sensillum and the reference electrode filled with the same Ringer solution was placed in the eye or the clypeus (for at 4 recordings). For recordings performed with a tungsten electrode, a tungsten rod $(0.01 \times 3$ inch, 717000, A-M Systems) secured in an electrode holder (ST50-BNC, Syskiyou) was sharpened in $0.5 \mathrm{~N} \mathrm{NaOH}$ with a microelectrode etcher (EE-1D, Bak Electronics) at $24 \mathrm{~V}$ for nine cycles. No more than three sensilla from the same antenna were recorded. All measurements were taken from distinct neurons except for recordings performed using the bridged configuration shown in Fig. $1 \mathrm{~b}$ (top and middle panels) and Supplementary Fig. 1.

AC signals $(100-20 \mathrm{k} \mathrm{Hz})$ and DC signals were simultaneously recorded on an NPI EXT-02F amplifier (ALA Scientific Instruments) and digitized at $5 \mathrm{kHz}$ with Digidata 1550 (Molecular Devices). ORN spikes were detected and sorted using threshold search under Event Detection in Clampfit 10 (Molecular Devices). Spike timing data were exported and analyzed in Igor Pro 6.3 (Wavemetrics). Peristimulus time histograms were obtained by averaging spike activities in 50 -ms bins and smoothed using a binomial filter (Igor Pro 6.3, Wavemetrics).

Sensillum types were identified based on their locations on the antenna or maxillary palp, and their characteristic odor response profiles ${ }^{15,21}$. For ac3I and ac3II sensilla, sensillum types were determined according to ORN-specific fluorescent labeling (ac3I: $\operatorname{Ir} 75 b$-GAL4; ac3II: $\operatorname{Ir} 75 c$-GAL4) because the response profiles for ac3I and ac3II in D. melanogaster are virtually indistinguishable 27 . Based on the location of the fluorescence signals, we recorded from ac3I with a medial mounting position and ac3II with a posterior mounting position ${ }^{55}$

Odor stimuli. Chemicals were $>99 \%$ pure or of the highest purity available at Sigma-Aldrich unless otherwise specified. Odorants were diluted in paraffin oil unless otherwise noted. Apple cider vinegar (Spectrum, naturals filtered apple cider vinegar) and geosmin were diluted in water, and trans-palmitoleic acid (Cayman Chemical) was diluted in ethanol. For odor mixture experiments, individual odorants $(2 \times$ stock solutions) were mixed either with paraffin oil or with another odorant at $1: 1(\mathrm{v} / \mathrm{v})$ ratio prior to experiments. For short odor pulses, odor stimuli ( $100 \mu \mathrm{l}$ applied to a filter disc) were delivered from a Pasteur pipette via a pulse of air $\left(200 \mathrm{ml} \mathrm{min}^{-1}\right)$ into the main air stream $\left(2000 \mathrm{ml} \mathrm{min}^{-1}\right)$. A Pasteur pipette filled with pure (Fig. 1 and Supplementary Fig. 1) or diluted $\mathrm{CO}_{2}\left(5 \times 10^{-2}\right.$, v/v in air, Supplementary Fig. 6) was used to deliver $\mathrm{CO}_{2}$ stimuli into the main air stream.
Background odor stimuli were delivered from a $125-\mathrm{ml}$ flask containing $3 \mathrm{ml}$ of odor dilutions directly downstream of the main air stream $\left(2000 \mathrm{ml} \mathrm{min}^{-1}\right)$.

For palmitoleic acid, $4.5 \mu \mathrm{l}$ of the freshly diluted odorant was applied to filter paper inserted inside a truncated $200-\mu$ l pipette tip. Ethanol was allowed to evaporate for $1 \mathrm{~h}$ in a vacuum desiccator prior to experiments. The odor cartridge was positioned around $4 \mathrm{~mm}$ away from the antenna as described ${ }^{54}$. Odor stimulus was delivered via a $500-\mathrm{ms}$ pulse of air $\left(500 \mathrm{ml} \mathrm{min}^{-1}\right)$ directly at the antenna in the presence of humidified air flow at $2000 \mathrm{mlmin}^{-1}$ from a different direction. Of note, female at $4 \mathrm{~A}$ does not respond to palmitoleic acid as strongly as male at $4 \mathrm{~A}$ in 7-day-old flies (Ng et al., unpublished data).

Optogenetic stimulation. Newly eclosed female flies expressing the H134R-ChR2 transgene in target ORNs were reared in constant darkness for 5 days on fly food supplemented with $100 \mu \mathrm{M}$ all trans-retinal (Sigma) unless otherwise specified. Flies were transferred to fresh retinal food 1 day prior to experiments. A light stimulus was generated via a blue LED $(470 \mathrm{~nm}$, Universal LED Illumination System, pE-4000, Cool LED). Light pulses (500-ms duration) were controlled by a shutter (Vincent Associates) driven by Clampex 10.4 (Molecular Devices). Light output around the position of the recorded antenna was measured with an optical power meter (PMKIT-05-01, Newport Corporation) via a slim profile wand detector (818-ST2/DB, Newport Corporation).

The LFP light responses in the ac3 neurons expressing H134R-ChR2 were too small to be reliably analyzed. Attempts to express H134R-ChR2 in ab5A (Or82aGAL4) or ab5B (Or47a-GAL4) failed to yield any light responses, despite the observation that the fluorescence of mCherry tag on H134-ChR2 was visible in the target ORNs. Aging the transgenic flies to 14 days old or increasing retinal concentrations in the food did not improve the situation.

Immunohistochemistry. Seven-day-old female files expressing myc-tagged Orco and/or Or85a odorant receptors in the ab2B ORNs were anesthetized on ice, with their heads aligned in a collar, covered with Cryo-OCT (Tissue-Tek, Fisher Scientific), and frozen on dry ice ${ }^{56}$. Cryosections $(14 \mu \mathrm{m})$ were fixed with $4 \%$ paraformaldehyde in $1 \times$ phosphate-buffered saline and stained with rabbit anti-myc antibody (1:250, 71D10, Cell Signaling Technology) and 21A6 (ciliary base marker, 1:200, DSHB), followed by goat anti-rabbit Alexa 647 secondary antibody (1:250, A21236, Life Technologies) and goat anti-mouse Alexa 568 (1:200, A11019, Life Technologies). Confocal microscopy was performed with a Zeiss 880 Airyscan Microscope, and images were processed with ImageJ software.

Sample preparation for SBEM. Target expression of APEX2 in ORNs for SBEM was performed as follows ${ }^{46}$. Briefly, transgenic Drosophila lines (10xUAS-mycAPEX2-Orco or 10xUAS-mCD8GF-APEX2) were generated to facilitate dendritic targeting of APEX2 ${ }^{46}$. Expression of APEX2 in select ORNs was driven by OrXGAL4 drivers (Supplementary Data 1). Six- to eight-day-old female flies were cold anesthetized prior to the dissection of their antennae. The antennae were processed with the CryoChem method ${ }^{46}$, which involves cryofixation by high-pressure freezing, freeze-substitution, rehydration, DAB labeling reaction, en bloc heavy metal staining, dehydration, and resin infiltration.

Microcomputed X-ray tomography was performed on resin-embedded specimens using a Versa $510 \mathrm{X}$-ray microscope (Zeiss) to determine DAB-labeled region of interest. The specimens were then mounted on aluminum pins with conductive silver epoxy (Ted Pella) and sputter coated with gold-palladium for SBEM imaging. The ab3, ab4, ac3II, at4 datasets were collected with a Gemini SEM (Zeiss) equipped with a 3 View block-face unit (Gatan); the ab5 dataset was collected with a Merlin scanning electron microscope (Zeiss) equipped with a 3View2XP and OnPoint backscatter detector (Gatan). Parameters for SBEM image acquisition are listed in Supplementary Table 5.

Segmentation of DAB-labeled Drosophila ORNs. The DAB-labeled Drosophila ORN was segmented in a semi-automated fashion using the IMOD software ${ }^{57}$ to 
generate a 3D model ${ }^{46}$. The IMOD command line "imodauto" was used for the auto-segmentation by setting thresholds to isolate the labeled neuron of interest. Auto-segmentation was followed by manual proofreading and correction of errors by two independent proofreaders. The neighboring, unlabeled ORN was manually segmented using the same software. Due to insufficient DAB labeling, the fine outer dendritic branches of most basiconic ORNs could not be reliably identified for segmentation.

Morphometric analysis. The 3D model of each ORN was first separated into cell body, inner dendrite and outer (sensory) dendrite models. The inner and outer dendrites were separated at the cilium base, a notably constricted dendritic region ${ }^{42}$. The volume measurements of ORNs were then obtained with the "imodinfo" function in IMOD based on the 3D models.

The lengths of most inner and outer dendrites were determined by first converting the 3D models into binary image files using the IMOD command "imodmop". Then the skeletons of the 3D images were extracted using Skeletonize3D (https://imagej.net/Skeletonize3D) plugin in Fiji (NIH). The lengths of the resulting skeletons were obtained by the Fiji "Analyze Skeleton" function. For the ab3A, ab4A and ab5A ORNs, which exhibit significant dendritic enlargements (Fig. 6f), their inner dendritic lengths were determined by first visually identifying the center point in every ninth contour of the 3D models (300 400 nm z-step), then manually measuring and summing the distances between those points. The inner dendritic lengths of the ab3B, ab4B, and ab5B ORNs were determined in the same way. The outer dendritic lengths reported in Supplementary Table 2 are the distances between the cilium bases and the tips of the longest dendritic branch.

The outer dendrites of ORNs were assumed to be cylindrical and their surface areas were calculated based on the measured volumes and lengths accordingly. The surface areas of cell bodies were measured with the "imodinfo" function in IMOD.

Statistics. All data presented as mean \pm s.e.m. were analyzed using Igor Pro 6.3 or SigmaPlot 13.0. Coefficients and the standard deviations of the linear fits were generated in Igor Pro 6.3. Unpaired two-tailed $t$ test was performed in Supplementary Fig. 8 for single variable comparison between two groups. Paired twotailed $t$ test was performed in Fig. 6 for the morphological comparison between grouped ORNs. Data are presented as mean \pm s.e.m. $P<0.05$ was considered to be statistically significant and is presented as ${ }^{*} P<0.05,{ }^{* *} P<0.01$, or ${ }^{* * *} P<0.001$. Statistical significance for linear coefficients was determined by analysis of covariance (ANCOVA) in RStudio using functions within the car package (version3.00 ). Data are presented as coefficient \pm s.d. $P<0.05$ was considered to be statistically significant and the differences are denoted by different letters.

Modeling. We consider the passive electric circuit model containing an auxiliary cell (denoted "A") and two ORNs $\left(\mathrm{ORN}_{1}\right.$ and $\left.\mathrm{ORN}_{2}\right)(\mathrm{Fig} .7 \mathrm{a})$. Each cell is modeled as an effective Thevenin circuit with battery and resistances. The auxiliary cell is modeled as a battery $E_{\mathrm{A}}$ in line with an input resistance $R_{\mathrm{A}}$. The soma of $\mathrm{ORN}_{1}$ is modeled as a battery $E_{1}$ with input resistance $R_{\text {in }}$, and the sensory dendrite is modeled as an odorant stimulation-dependent resistance $R_{\mathrm{d} 1}$ (similar for $\mathrm{ORN}_{2}$ ). The driving forces for the currents are the batteries $E_{\mathrm{A}}, E_{1}$ and $E_{2}$. The voltage $V_{\mathrm{A}}$ is the transepithelium potential, and $V_{\mathrm{m} 1}$ and $V_{\mathrm{m} 2}$ are the neuronal transmembrane potentials that control spike firing. Changes in $V_{\mathrm{A}}$ are recorded as changes in the local field potential, LFP. Of note, this passive electric circuit model concerns voltage changes resulting from constant odor stimulation. Therefore, all capacitive currents are considered zero in this model.

The system of equations for the currents $I_{\mathrm{A}}, I_{1}$ and $I_{2}$ reads

$$
\begin{gathered}
V_{\mathrm{A}}=E_{\mathrm{A}}+I_{\mathrm{A}} R_{\mathrm{A}}, \\
V_{\mathrm{A}}=E_{1}+I_{1} R_{1}, \\
V_{\mathrm{A}}=E_{2}+I_{2} R_{2}, \\
0=I_{\mathrm{A}}+I_{1}+I_{2},
\end{gathered}
$$

where we introduce the total resistances $R_{1}=R_{\mathrm{in} 1}+R_{\mathrm{d} 1}$ and $R_{2}=R_{\mathrm{in} 2}+R_{\mathrm{d} 2}$. By solving these equations, we obtain the currents

$$
\begin{aligned}
& I_{\mathrm{A}}=\frac{E_{1} \frac{R_{2}}{R_{1}+R_{2}}+E_{2} \frac{R_{1}}{R_{1}+R_{2}}-E_{\mathrm{A}}}{\frac{R_{1} R_{2}}{R_{1} R_{2}+R_{\mathrm{A}}},}, \\
& I_{1}=\frac{E_{2}-E_{1}}{R_{1}+R_{2}}-I_{\mathrm{A}} \frac{R_{2}}{R_{1}+R_{2}}, \\
& I_{2}=\frac{E_{1}-E_{2}}{R_{1}+R_{2}}-I_{\mathrm{A}} \frac{R_{1}}{R_{1}+R_{2}} .
\end{aligned}
$$

The neuronal part is an effective Thevenin circuit with battery $E_{\mathrm{T}}=E_{1} \frac{R_{2}}{R_{1}+R_{2}}+$ $E_{2} \frac{R_{1}}{R_{1}+R_{2}}$ and resistance $R_{\mathrm{T}}=\frac{R_{1} R_{2}}{R_{1}+R_{2}}$. The neuronal membrane potentials are

$$
\begin{aligned}
& V_{\mathrm{m} 1}=E_{1}+R_{\mathrm{in} 1} I_{1}, \\
& V_{\mathrm{m} 2}=E_{2}+R_{\mathrm{in} 2} I_{2} .
\end{aligned}
$$

To further simplify the analysis, we use $R_{\mathrm{A}}$ to define dimensionless resistances $\tilde{R}_{\mathrm{A}}=R_{\mathrm{A}} / R_{\mathrm{A}}=1, \tilde{R}_{1}=R_{1} / R_{\mathrm{A}}, \tilde{R}_{2}=R_{2} / R_{\mathrm{A}}$ and rescale currents

$\widetilde{I}_{\mathrm{A}}=I_{\mathrm{A}} R_{\mathrm{A}}, \widetilde{I}_{1}=I_{1} R_{\mathrm{A}}$ and $\tilde{I}_{2}=I_{2} R_{\mathrm{A}}$. This does not affect the voltages in Eqs. 1 and 3 and is formally equivalent to setting $R_{\mathrm{A}}=1$. In the following, we therefore omit the tilde symbols and simply use $R_{\mathrm{A}}=1$. As mentioned before, this does not affect the results and conclusions concerning voltages.

We next focus on the input and dendritic resistances. We are interested in how odorant stimulation and the morphometric properties of soma and sensory dendrites affect the transmembrane potentials $V_{\mathrm{m} 1}$ and $V_{\mathrm{m} 2}$. We assume that the input resistances $R_{\mathrm{in} 1}$ and $R_{\mathrm{in} 2}$ depend on the size of the soma and a smaller ORN has a higher input resistance compared to a larger ORN. Specifically, we assume that $R_{\mathrm{in} 1}$ and $R_{\mathrm{in} 2}$ are inversely proportional to the soma surfaces $A_{\mathrm{s} 1}$ and $A_{\mathrm{s} 2}$

$$
R_{\text {in } 1}=\frac{\rho_{\mathrm{s}}}{A_{\mathrm{s} 1}} \quad \text { and } \quad R_{\mathrm{in} 2}=\frac{\rho_{\mathrm{s}}}{A_{\mathrm{s} 2}}
$$

where $\rho_{\mathrm{s}}$ is the soma membrane resistivity.

We model the sensory dendrite of an ORN as a uniform cylinder of length $L$ with axial (cytoplasmic) resistivity $r_{\mathrm{a}}$ and membrane resistivity $r_{\mathrm{m}}$, where $r_{\mathrm{m}}$ depends on odor activation. From linear cable theory, the input resistance $R_{\mathrm{s}}$ of a uniform leaky cylinder with an open and a sealed end is

$$
R_{\mathrm{d}}=\sqrt{r_{\mathrm{m}} r_{\mathrm{a}}} \operatorname{coth}\left(L \sqrt{\frac{r_{\mathrm{a}}}{r_{\mathrm{m}}}}\right)
$$

where $l=L \sqrt{\frac{r_{\mathrm{a}}}{r_{\mathrm{m}}}}$ is the electrotonic length of the cylinder and $R_{\infty}=\sqrt{r_{\mathrm{m}} r_{\mathrm{a}}}$ is the input resistance of an infinitely long cylinder. We further simplify Eq. 5 assuming that the cytoplasmic resistance is much smaller than the membrane resistance such that $l \ll 1^{58}$. By expanding Eq. 5 for $l \ll 1$ we get in first order

$$
R_{\mathrm{d}}=\frac{r_{\mathrm{m}}}{L}=\frac{\rho_{\mathrm{d}}}{A_{\mathrm{d}}},
$$

where $\rho_{\mathrm{d}}$ is the dendritic membrane resistivity and $A_{\mathrm{d}}$ is the dendritic surface.

The membrane resistivity $\rho_{\mathrm{d}}$ implicitly depends on odor activation because odorants activate receptors that increase the membrane conductivity. We simplify the transduction process and assume that for a given stimulation with odorant concentration "od", the amount of activated receptors is given by the Hill function

$$
R^{*}=R_{\max }^{*} \frac{\mathrm{od}^{n}}{\mathrm{od}^{n}+K_{\mathrm{od}}^{n}}
$$

where $n$ is the Hill-coefficient and $K_{\text {od }}$ the odorant concentration that activates half of the receptors. Receptor activation induces the additional membrane conductivity $\sigma_{\mathrm{d}}=\gamma R^{*}=\sigma_{\mathrm{d}, \max } \frac{\mathrm{od}^{n}}{\mathrm{od}^{n}+K_{\mathrm{od}}^{n}}$. With the basal resistivity $\rho_{\mathrm{d}, 0}$, the overall conductivity is $\frac{1}{\rho_{\mathrm{d}}}=\frac{1}{\rho_{\mathrm{d}, 0}}+\sigma_{\mathrm{d}}=\frac{1+\rho_{\mathrm{d}, 0} \sigma_{\mathrm{d}}}{\rho_{\mathrm{d}, 0}}$. This gives

$$
\rho_{\mathrm{d}}=\frac{\rho_{\mathrm{d}, 0}}{1+g}
$$

with

$$
g=g_{\max } \frac{\mathrm{od}^{n}}{\mathrm{od}^{n}+K_{\mathrm{od}}^{n}}
$$

and $g_{\max }=\rho_{\mathrm{d}, 0} \sigma_{\mathrm{d}, \max }$

In summary, to model the dendritic resistances we use the formula

$$
R_{\mathrm{d}}=\frac{\rho_{\mathrm{d}, 0}}{A_{\mathrm{d}}(1+g)} .
$$

We next address the changes in local field potential due to stimulation of $\mathrm{ORN}_{1}$ or $\mathrm{ORN}_{2}$. When the activation of $\mathrm{ORN}_{1}$ is altered due to odorant stimulation, the dendritic resistance $R_{\mathrm{d} 1}$ changes by $\Delta R_{\mathrm{d} 1}$ which alters the local field potential LFP by $\left|\Delta V_{\mathrm{A}}\right|$. From Eqs. 1 to 3 we compute that the corresponding changes in the membrane potentials are $\Delta V_{\mathrm{m} 2}=\Delta V_{\mathrm{A}} \frac{R_{\mathrm{in} 2}}{R_{2}}$ and

$$
\Delta V_{\mathrm{m} 1}=-\Delta V_{\mathrm{A}} R_{\mathrm{in} 1}\left(1+\frac{1}{R_{2}}\right) .
$$

Similarly, when $\mathrm{ORN}_{2}$ is stimulated we have $\Delta V_{\mathrm{m} 1}=\Delta V_{\mathrm{A}} \frac{R_{\text {inl }}}{R_{1}}$ and

$$
\Delta V_{\mathrm{m} 2}=-\Delta V_{\mathrm{A}} R_{\mathrm{in} 2}\left(1+\frac{1}{R_{1}}\right) .
$$

Because $R_{2}\left(R_{1}\right)$ remains constant when $\mathrm{ORN}_{1}\left(\mathrm{ORN}_{2}\right)$ is stimulated ${ }^{12}$, it follows that $\Delta V_{\mathrm{m} 1}$ and $\Delta V_{\mathrm{m} 2}$ change linearly with $\Delta V_{\mathrm{A}}$. Assume that $\mathrm{ORN}_{1}$ corresponds to the larger neuron $\mathrm{A}$ and $\mathrm{ORN}_{2}$ to the smaller neuron $\mathrm{B}$ such that $R_{\text {in } 1}<R_{\text {in2 }}$ and $R_{1}<R_{2}$. With this we have $R_{\text {in1 }}\left(1+\frac{1}{R_{2}}\right)<R_{\text {in } 2}\left(1+\frac{1}{R_{1}}\right)$ which is in agreement with Fig. 3 showing that the slope of the spiking rate (which reflects $\Delta V_{\mathrm{m} 1}$ or $\left.\Delta V_{\mathrm{m} 2}\right)$ vs. LFP is smaller for $\mathrm{ORN}_{\mathrm{A}}$ compared to $\mathrm{ORN}_{\mathrm{B}}$.

The following sections concern the fitting procedure. We are interested in whether the differences between our electrophysiological measurements can be explained by the morphometric differences among the grouped ORNs. To address this question, we focus on $a b 3, a b 4$ and ab5 sensilla for which we have electrophysiological and morphometric data. We simultaneously fitted the combined LFP data (Fig. 2) using a minimal model with a common set of basic parameters and ORN-specific parameters (surface area and odorant sensitivity). In the following, we derive the minimal model that we use for the fitting procedure. For values of fitting parameters, see Tables 1-3. The fitting and simulation results are presented in Fig. 7 of the main text. 
We were able to fit the combined LFP measurements of ab3, ab4, and ab5 neurons by assuming a common set of parameters that differs only in the areas of soma and sensory dendrite. However, we could not adequately fit the combined LFP data of ac3II, ab3, ab4 and ab5 neurons by assuming such common set of parameters. We note that basiconic ORNs (ab3, ab4, ab5) have highly branched sensory dendrites, whereas coeloconic ORNs do not (ac3II). It is possible that the branching pattern of sensory dendrites or other unknown factors may have introduced additional parameters that affect the fitting results.

To estimate the batteries $E_{1}$ and $E_{2}$ using the resting membrane potential, we assume that the auxiliary cell is the same for all sensillum types, and therefore use a single value for $E_{\mathrm{A}}$. For $E_{1}$ and $E_{2}$, we do not assume that they are identical for all sensilla because ORN basal resistances differ depending on the morphometric feature of the neuron, and using the same $E_{1}$ and $E_{2}$ for all sensilla would lead to different resting membrane potentials for the ORNs (data not shown). This is problematic if one assumes that the mechanism of action potential generation is similar in all ORNs. Instead, we assume that at rest without odorant stimulation (basal condition) the membrane potentials of the ORNs are identical such that $V_{\mathrm{m} 1}$ $=V_{\mathrm{m} 2}=V_{0}$ with $V_{0}=-60 \mathrm{mV}$. We used the conditions $V_{\mathrm{m} 1}=V_{\mathrm{m} 2}=V_{0}$ to express the parameters $E_{1}$ and $E_{2}$ as a function of $V_{0}$. With Eqs. 2 and 3 we find

$$
\begin{aligned}
& E_{1}=E_{\mathrm{A}}-\left(E_{\mathrm{A}}-V_{0}\right) \Theta_{0}\left(1+\frac{\xi_{0}}{2}\right), \\
& E_{2}=E_{\mathrm{A}}-\left(E_{\mathrm{A}}-V_{0}\right) \Theta_{0}\left(1-\frac{\xi_{0}}{2}\right)
\end{aligned}
$$

and

$$
\begin{array}{r}
I_{\mathrm{A}}=-\left(E_{\mathrm{A}}-V_{0}\right) \Theta_{0}\left(\xi_{0}\left(\alpha_{1}-\alpha_{2}\right)+\beta_{1}+\beta_{2}\right), \\
I_{1}=\left(E_{\mathrm{A}}-V_{0}\right) \Theta_{0}\left(\xi_{0} \alpha_{1}+\beta_{1}\right), \\
I_{2}=\left(E_{\mathrm{A}}-V_{0}\right) \Theta_{0}\left(-\xi_{0} \alpha_{2}+\beta_{2}\right)
\end{array}
$$

\begin{tabular}{|c|c|c|c|}
\hline \multirow[t]{2}{*}{ ORN type } & \multicolumn{2}{|c|}{ Surface area $\left(\mu \mathrm{m}^{2}\right)$} & \multirow[b]{2}{*}{ Sensory dendrite } \\
\hline & & Soma & \\
\hline \multirow[t]{2}{*}{ ab3 } & $A$ & 98 & 46 \\
\hline & B & 91 & 16 \\
\hline \multirow[t]{2}{*}{$a b 4$} & A & 137 & 38 \\
\hline & B & 75 & 25 \\
\hline \multirow[t]{2}{*}{ ab5 } & $A$ & 65 & 20 \\
\hline & B & 67 & 20 \\
\hline
\end{tabular}

Table 1 ORN-specific morphometric parameters

\section{Table 2 ORN-specific odorant sensitivities}

\begin{tabular}{lll} 
ORN type & & $\boldsymbol{k}_{\text {od }}$ \\
\hline $\mathrm{ab3}$ & $\mathrm{A}$ & -4.8 \\
& $\mathrm{~B}$ & -5.1 \\
$\mathrm{ab} 4$ & $\mathrm{~A}$ & -5.9 \\
& $\mathrm{~B}$ & -2.5 \\
$\mathrm{ab5}$ & $\mathrm{A}$ & -1 \\
& $\mathrm{~B}$ & -2 \\
\hline
\end{tabular}

\section{Table 3 Common parameters}

\section{Parameters Description}

$E_{\mathrm{A}}(\mathrm{mV})$

$V_{0}(\mathrm{mV})$

$\rho_{\mathrm{s}}\left(\mu \mathrm{m}^{2}\right)$

$\rho_{\mathrm{d}, \mathrm{O}}\left(\mu \mathrm{m}^{2}\right)$

$n$

$g_{\max }$

\section{Auxiliary cell battery}

ORN resting membrane potential

ORN soma membrane resistivity, rescaled by $R_{A}$

ORN sensory dendrite basal membrane 17

resistivity, rescaled by $R_{\mathrm{A}}$

ORN Hill-coefficient for odorant activation

$=\rho_{\mathrm{d}, 0} \sigma_{\mathrm{d}, \max }$. Where $\sigma_{\mathrm{d}, \max }$ is the maximum dendritic membrane conductivity induced by receptor activation with

$$
\begin{aligned}
& \alpha_{1}=\frac{1+\frac{R_{2}}{2}}{R_{1} R_{2}+R_{1}+R_{2}}, \\
& \beta_{1}=\frac{R_{2}}{R_{1} R_{2}+R_{1}+R_{2}}, \\
& \alpha_{2}=\frac{1+\frac{R_{1}}{2}}{R_{1} R_{2}+R_{1}+R_{2}}, \\
& \beta_{2}=\frac{R_{1}}{R_{1} R_{2}+R_{1}+R_{2}}
\end{aligned}
$$

and

$$
\begin{aligned}
\xi_{0} & = \\
\Theta_{0} & =\frac{\frac{R_{\text {in }} \beta_{1,0}-R_{\text {in } 2} \beta_{2,0}}{1-R_{\text {in } 1} \alpha_{1,0}-R_{\text {in } 2} \alpha_{2,0}},}{1-\frac{1}{2}\left(R_{\text {in } 1}\left(\xi_{0} \alpha_{1,0}+\beta_{1,0}\right)+R_{\text {in } 2}\left(-\xi_{0} \alpha_{2,0}+\beta_{2,0}\right)\right)} .
\end{aligned}
$$

The values $\beta_{1,0}, \beta_{2,0}, \alpha_{1,0}$ and $\alpha_{2,0}$ are obtained from Eq. 15 by inserting basal dendritic resistances that depend on the neuronal morphometry. Equation 14 shows that the effective driving force for the currents is $E_{\mathrm{A}}-V_{0}$.

Next we use the spike/LFP ratio to constrain the parameter space. For the ab5 sensilla, we use the measured values for the outer dendritic surfaces $A_{\mathrm{d} 1}$ and $A_{\mathrm{d} 2}$. In contrast, for ab3 and ab4, the surfaces $A_{\mathrm{d} 1}$ and $A_{\mathrm{d} 2}$ are unavailable and are therefore free fitting parameters. However, for these sensilla, we use the measured spike/LFP ratio $r$ (Fig. 3) to further constrain the parameter space. Our passive electrical model does not predict spiking rates. However, we assume that $\Delta V_{\mathrm{m} 1} /$ $\Delta V_{\mathrm{A}}$ or $\Delta V_{\mathrm{m} 2} / \Delta V_{\mathrm{A}}$ is correlated to the spike/LFP ratio shown in Fig. 3. Let $r>1$ be the ratio of the spike/LFP slopes of $\mathrm{ORN}_{\mathrm{A}}$ to $\mathrm{ORN}_{\mathrm{B}}$ shown in Fig. 3 . With the convention that $\mathrm{ORN}_{1}$ corresponds to $\mathrm{ORN}_{\mathrm{A}}$ and $\mathrm{ORN}_{2}$ to $\mathrm{ORN}_{\mathrm{B}}$, we have

$$
r=\frac{R_{\mathrm{in} 2}\left(1+\frac{1}{R_{1,0}}\right)}{R_{\mathrm{in} 1}\left(1+\frac{1}{R_{2,0}}\right)},
$$

where $R_{1,0}$ and $R_{2,0}$ are basal resistances without odorant stimulation. We can use Eq. 17 to express $R_{1,0}$ as a function of $R_{2,0}$

$$
R_{1,0}=\frac{R_{2,0}}{r \kappa+R_{2,0}(r \kappa-1)},
$$

where $\kappa=\frac{R_{\mathrm{in} 1}}{R_{\mathrm{in} 2}}$. With $R_{1,0}=R_{\mathrm{d} 1,0}+R_{\mathrm{in} 1}=\frac{\rho_{\mathrm{d}, 0}}{A_{\mathrm{d} 1}}+R_{\mathrm{in} 1}$ the dendritic surface of $\mathrm{ORN}_{1}$ is

$$
A_{\mathrm{d} 1}=\rho_{\mathrm{d}, 0}\left(\frac{R_{2,0}}{r \kappa+R_{2,0}(r \kappa-1)}-R_{\mathrm{in} 1}\right)^{-1} .
$$

In summary, for ab3 and ab4, we use $A_{\mathrm{d} 2}$ and $r$ as fitting parameters and $A_{\mathrm{d} 1}$ is computed with Eq. 19. The range for $r$ is constrained by the values shown in Fig. 3 . In contrast, for ab5 we use our measured values for $A_{\mathrm{d} 1}$ and $A_{\mathrm{d} 2}$.

For the odorant concentration, we write od $=c_{0} 10^{x}$, where $x<0$ corresponds to the odorant dilution and $c_{0}$ is the initial undiluted concentration. By writing $K_{\mathrm{od}}=$ $c_{0} 10^{k_{\text {od }}}$ we have

$$
\frac{\mathrm{od}^{n}}{\mathrm{od}^{n}+K_{\mathrm{od}}^{n}}=\frac{1}{1+10^{n\left(k_{\mathrm{od}}-x\right)}} .
$$

The parameter $K_{\text {od }}$ (resp. $k_{\text {od }}$ ) characterizes the sensitivity of the ORN to the odorant and is specific for each ORN. We therefore allow for each neuron a different parameter $k_{\text {od }}$

Tables 1-3 indicate the parameters for the electric circuit model.

Animal research. We have complied with all relevant ethical regulations for animal testing and research. No specific ethical approval is required for this study.

\section{Data availability}

The data that support the findings of this study are available from the corresponding author upon request. The source data underlying Figs. 2, 3, 4, 5, 6, and 7b-f and Supplementary Figs. 1, 3, 5, 6, 7, 8 and 10 are provided as a Source Data file.

Received: 21 August 2018 Accepted: 4 March 2019

Published online: 05 April 2019

\section{References}

1. Buzsáki, G., Anastassiou, C. A. \& Koch, C. The origin of extracellular fields and currents-EEG, ECoG, LFP and spikes. Nat. Rev. Neurosci. 13, 407-420 (2012)

2. Jefferys, J. G. R. Nonsynaptic modulation of neuronal activity in the brain: electric currents and extracellular ions. Physiol. Rev. 75, 689-723 (1995). 
3. Faber, D. S. \& Korn, H. Electrical field effects: their relevance in central neural networks. Physiol. Rev. 69, 821-863 (1989).

4. Arvanitaki, A. Effects evoked in an axon by the activity of a contiguous one. J. Neurophysiol. 5, 89-108 (1942).

5. Katz, B. \& Schmitt, O. H. Electric interaction between two adjacent nerve fibres. J. Physiol. 97, 471-488 (1940).

6. Bokil, H., Laaris, N., Blinder, K., Ennis, M. \& Keller, A. Ephaptic interactions in the mammalian olfactory system. J. Neurosci. 21, RC173 (2001).

7. Damasio, A. \& Carvalho, G. B. The nature of feelings: evolutionary and neurobiological origins. Nat. Rev. Neurosci. 14, 143-152 (2013).

8. Kamermans, M. Hemichannel-mediated inhibition in the outer retina. Science 292, 1178-1180 (2001).

9. Han, K. et al. Ephaptic coupling promotes synchronous firing of cerebellar Purkinje cells. Neuron 100, 564-578 (2018)

10. Hubel, D. H. Cortical neurobiology. Annu. Rev. Neurosci. 5, 363-370 (1982).

11. Su, C.-Y., Menuz, K., Reisert, J. \& Carlson, J. R. Non-synaptic inhibition between grouped neurons in an olfactory circuit. Nature 492, 66-71 (2012).

12. Vermeulen, A. \& Rospars, J.-P. Why are insect olfactory receptor neurons grouped into sensilla? The teachings of a model investigating the effects of the electrical interaction between neurons on the transepithelial potential and the neuronal transmembrane potential. Eur. Biophys. J. 33, 633-643 (2004).

13. Redkozubov, A. High electrical resistance of the bombykol cell in an olfactory sensillum of Bombyx mori: voltage- and current-clamp analysis. J. Insect Physiol. 41, 451-455 (1995).

14. de Bruyne, M., Clyne, P. J. \& Carlson, J. R. Odor coding in a model olfactory organ: the Drosophila maxillary palp. J. Neurosci. 19, 4520-4532 (1999).

15. de Bruyne, M., Foster, K. \& Carlson, J. R. Odor coding in the Drosophila antenna. Neuron 30, 537-552 (2001).

16. Hallem, E. A., Ho, M. G. \& Carlson, J. R. The molecular basis of odor coding in the Drosophila antenna. Cell 117, 965-979 (2004).

17. Fishilevich, E. \& Vosshall, L. B. Genetic and functional subdivision of the Drosophila antennal lobe. Curr. Biol. 15, 1548-1553 (2005).

18. Couto, A., Alenius, M. \& Dickson, B. J. Molecular, anatomical, and functional organization of the Drosophila olfactory system. Curr. Biol. 15, 1535-1547 (2005).

19. Linz, J. et al. Host plant-driven sensory specialization in Drosophila erecta. Proc. Biol. Sci. 280, 20130626 (2013).

20. Stensmyr, M. C., Dekker, T. \& Hansson, B. S. Evolution of the olfactory code in the Drosophila melanogaster subgroup. Proc. Biol. Sci. 270, 2333-2340 (2003).

21. Hallem, E. A. \& Carlson, J. R. Coding of odors by a receptor repertoire. Cell 125, 143-160 (2006).

22. Denk, W. \& Horstmann, H. Serial block-face scanning electron microscopy to reconstruct three-dimensional tissue nanostructure. PLoS Biol. 2, e329 (2004).

23. van der Goes van Naters, W. Inhibition among olfactory receptor neurons. Front. Hum. Neurosci. 7, 690 (2013).

24. Jones, W. D., Cayirlioglu, P., Kadow, I. G. \& Vosshall, L. B. Two chemosensory receptors together mediate carbon dioxide detection in Drosophila. Nature 445, 86-90 (2007).

25. Jeong, Y. et al. An odorant-binding protein required for suppression of sweet taste by bitter chemicals. Neuron 79, 725-737 (2013).

26. Su, C.-Y., Menuz, K. \& Carlson, J. R. Olfactory perception: receptors, cells, and circuits. Cell 139, 45-59 (2009).

27. Prieto-Godino, L. L. et al. Evolution of acid-sensing olfactory circuits in Drosophilids. Neuron 93, 661-676 (2017).

28. Nagel, K. I. \& Wilson, R. I. Biophysical mechanisms underlying olfactory receptor neuron dynamics. Nat. Neurosci. 14, 208-216 (2011).

29. Guillet, J. C. \& Bernard, J. Shape and amplitude of the spikes induced by natural or electrical stimulation in insect receptors. J. Insect Physiol. 18, 2155-2171 (1972).

30. Bernard, J. \& Guillet, J. C. Changes in the receptor potential under polarizing currents in two insect receptors. J. Insect Physiol. 18, 2173-2187 (1972).

31. Kaissling, K. E. \& Thorson, J. Insect olfactory sensilla: structure, chemical and electrical aspect of the functional organization. Receptors for Neurotransmitters, Hormones, and Pheromones in Insects: Proceedings of the Workshop on Neurotransmitter and Hormone Receptors in Insects held in Cambridge (1980).

32. Martelli, C., Carlson, J. R. \& Emonet, T. Intensity invariant dynamics and odor-specific latencies in olfactory receptor neuron response. J. Neurosci. 33, 6285-6297 (2013).

33. Pulver, S. R., Pashkovski, S. L., Hornstein, N. J., Garrity, Pa \& Griffith, L. C. Temporal dynamics of neuronal activation by Channelrhodopsin-2 and TRPA1 determine behavioral output in Drosophila larvae. J. Neurophysiol. 101, 3075-3088 (2009).

34. Ronderos, D. S., Lin, C.-C., Potter, C. J. \& Smith, D. P. Farnesol-detecting olfactory neurons in Drosophila. J. Neurosci. 34, 3959-3968 (2014).

35. Larsson, M. C. et al. Or83b encodes a broadly expressed odorant receptor essential for Drosophila olfaction. Neuron 43, 703-714 (2004).
36. Benton, R., Vannice, K. S., Gomez-Diaz, C. \& Vosshall, L. B. Variant ionotropic glutamate receptors as chemosensory receptors in Drosophila. Cell 136, 149-162 (2009).

37. Wicher, D. et al. Drosophila odorant receptors are both ligand-gated and cyclic-nucleotide-activated cation channels. Nature 452, 1007-1011 (2008)

38. Sato, K. et al. Insect olfactory receptors are heteromeric ligand-gated ion channels. Nature 452, 1002-1006 (2008).

39. Butterwick, J. A. et al. Cryo-EM structure of the insect olfactory receptor Orco. Nature 560, 447-452 (2018)

40. Lin, C.-C., Prokop-Prigge, K. A., Preti, G. \& Potter, C. J. Food odors trigger Drosophila males to deposit a pheromone that guides aggregation and female oviposition decisions. eLife 4, e08688 (2015).

41. Stensmyr, M. C. et al. A conserved dedicated olfactory circuit for detecting harmful microbes in Drosophila. Cell 151, 1345-1357 (2012).

42. Shanbhag, S. R., Muller, B. \& Steinbrecht, R. A. Atlas of olfactory organs of Drosophila melanogaster 2. Internal organization and cellular architecture of olfactory sensilla. Arthropod Struct. Dev. 29, 211-229 (2000).

43. Keil, T. A. Reconstruction and morphometry of silkmoth olfactory hairs: a comparative study of sensilla trichodea on the antennae of male Antheraea polyphemus and Antheraea pernyi (Insecta, Lepidoptera). Zoomorphology 104, 147-156 (1984).

44. Hansson, B. S., Hallberg, E., Lofstedt, C. \& Steinbrecht, R. A. Correlation between dendrite diameter and action potential amplitude in sex pheromone specific receptor neurons in male Ostrinia nubilalis (Lepidoptera: pyralidae). Tissue Cell 26, 503-512 (1994).

45. Gnatzy, W., Mohren, W. \& Steinbrecht, R. A. Pheromone receptors in Bombyx mori and Antheraea pernyi-II. Morphometric analysis. Cell Tissue Res. 235, 35-42 (1984).

46. Tsang, T. K. et al. High-quality ultrastructural preservation using cryofixation for 3D electron microscopy of genetically labeled tissues. ELife 7, e35524 (2018).

47. Lam, S. S. et al. Directed evolution of APEX2 for electron microscopy and proximity labeling. Nat. Methods 12, 51-54 (2015).

48. Raue, A. et al. Data2Dynamics: a modeling environment tailored to parameter estimation in dynamical systems. Bioinformatics 31, 3558-3560 (2015).

49. Shimizu, K. \& Stopfer, M. Intimate neuronal whispers. Nature 492, 44-45 (2012).

50. Pettersen, K. H. \& Einevoll, G. T. Amplitude variability and extracellular lowpass filtering of neuronal spikes. Biophys. J. 94, 784-802 (2008).

51. Schnaitmann, C. et al. Color processing in the early visual system of Drosophila. Cell 172, 318-330 (2018)

52. Takemura, S. et al. Synaptic circuits and their variations within different columns in the visual system of Drosophila. Proc. Natl. Acad. Sci. USA 112, 13711-13716 (2015)

53. Miriyala, A., Kessler, S., Rind, F. C. \& Wright, G. A. Burst firing in bee gustatory neurons prevents adaptation. Curr. Biol. 28, 1585-1594 (2018).

54. Ng, R., Lin, H.-H., Wang, J. W. \& Su, C.-Y. Electrophysiological recording from Drosophila trichoid sensilla in response to odorants of low volatility. J. Vis. Exp. e56147, 1-8 (2017).

55. Lin, C. C. \& Potter, C. J. Re-classification of Drosophila melanogaster trichoid and intermediate sensilla using fluorescence-guided single sensillum recording. PLoS ONE 10, e0139675 (2015).

56. Saina, M. \& Benton, R. Visualizing olfactory receptor expression and localization in Drosophila. Methods Mol. Biol. 1003, 211-228 (2013).

57. Kremer, J. R., Mastronarde, D. N. \& McIntosh, J. R. Computer visualization of three-dimensional image data using IMOD. J. Struct. Biol. 116, 71-76 (1996).

58. Koch, C. Biophysics of Computation (Oxford University Press, Oxford, UK, 2004).

\section{Acknowledgements}

We thank Jeffry Isaacson, Johannes Reisert, Jing Wang, Larry Squire, Gareth Thomas and Renny Ng for comments on the manuscript; Szu-Ying Chen, Vivian Nguyen, Renny Ng, Edie Zhang, Martin Orden, Uma Talagadadivi, Ben Damasco, Tiffany Tsai and Cesar Navagonzales for assistance in ORN segmentations; Cesar Nava-gonzales for assistance in the analysis of natural odors. This work was supported by Frontiers of Innovation Scholars Program and Croucher Foundation Scholarship to T.K.T.; Hellman Fellowship, the Ray Thomas Edwards Foundation Career Development Award, Kavli Institute for Brain and Mind Innovative Research Grant and NIH R01DC015519 to C.-Y.S. National Center for Microscopy and Imaging Research (NCMIR) technologies and instrumentation are supported by grant GM103412 from the National Institute of General Medical Sciences.

\section{Author contributions}

Y.Z., T.K.T., and C.-Y.S. designed the experiments. Y.Z. performed sensillum recordings, data analysis, and natural odor analysis. T.K.T. and E.A.B. performed SBEM imaging with support from M.H.E. T.K.T. performed immunostaining and morphometric analysis. L.-A.C. conducted preliminary morphometric analysis using super-resolution confocal microscopy with support from A.-S.C. J.R. performed computational modeling. Y.Z., T.K.T., J.R., and C.-Y.S. wrote the manuscript with inputs from all authors. 


\section{Additional information}

Supplementary Information accompanies this paper at https://doi.org/10.1038/s41467019-09346-z.

Competing interests: The authors declare no competing interests.

Reprints and permission information is available online at http://npg.nature.com/ reprintsandpermissions/

Journal peer review information: Nature Communications thanks the anonymous reviewers for their contribution to the peer review of this work.

Publisher's note: Springer Nature remains neutral with regard to jurisdictional claims in published maps and institutional affiliations. (c) Open Access This article is licensed under a Creative Commons Attribution 4.0 International License, which permits use, sharing, adaptation, distribution and reproduction in any medium or format, as long as you give appropriate credit to the original author(s) and the source, provide a link to the Creative Commons license, and indicate if changes were made. The images or other third party material in this article are included in the article's Creative Commons license, unless indicated otherwise in a credit line to the material. If material is not included in the article's Creative Commons license and your intended use is not permitted by statutory regulation or exceeds the permitted use, you will need to obtain permission directly from the copyright holder. To view a copy of this license, visit http://creativecommons.org/ licenses/by/4.0/.

(C) The Author(s) 2019 